D.T. LE AND M. OKA

KODAI MATH. J.

18 (1995), $1-36$

\title{
ON RESOLUTION COMPLEXITY OF PLANE CURVES
}

\author{
LÊ DŨNG TRÁNG AND MUTSUO OKA
}

\section{Introduction}

The embedded resolution of a plane curve is a known process which was already known by Max Noether in $[\mathrm{N}]$. One may find an elementary account of this process in the book of E. Brieskorn and H. Knörrer [B-K].

When the plane curve is locally irreducible, it is easily observed that the case of one Puiseux pair is solved by only one good toroidal blowing-up. More generally with one good toroidal blowing-up one may eliminate the first Puiseux pair. Therefore after $g$ good toroidal blowing-ups, one can solve a curve singularity with one branch and $g$ Puiseux pairs.

In this paper we show that this phenomenon is general. Namely we prove that the minimal number of required toroidal blowing-ups to resolve the curve singularity is a topological invariant of the singularity that we have called the complexity of the resolution (see Theorem (3.12)).

In fact the complexity of a plane curve singularity is expected to behave like a depth. If we expect that a non-degenerate hypersurface singularity has resolution complexity one, it is reasonable to prove that a general plane section of that hypersurface is a curve which singularity has complexity at most equal to the dimension of the hypersurface. This result was actually proved by the second author in [O4].

It remains to understand what is the resolution complexity of a hypersurface singularity. We hope that this paper will draw the interest of the specialists on this subject.

\section{Choice of good coordinates}

Let $f(x, y)$ be a complex analytic function of two variables defined on an open neighborhood $U$ of the origin $O$ of $C^{2}$ and suppose that $f(O)=0$. Let

$$
f(x, y)=\Sigma c_{\alpha, \beta} x^{\alpha} y^{\beta}
$$

be the Taylor expansion of $f$ at the origin $O=(0,0)$. The Newton polygon

This research is done with the help of University of Paris VlI, Tokyo Institute of Technology and URA 212 of CNRS.

Received September 24, 1993. 
$\Gamma_{+}(f ; x, y)$ of $f$ at the point $O$ relatively to the coordinate system $(x, y)$ is the convex closure of the set

$$
\boldsymbol{c}_{\alpha, \beta} \neq 0
$$

The Newton boundary $\Gamma(f ; x, y)$ of $f$ at $O$ is the union of compact faces of the boundary of the Newton polygon of $f$ at $O$. Notice that the boundary of the Newton polygon differs from the Newton boundary by two non-compact faces parallel to the coordinate axes. For each compact face $\Delta$ of $\Gamma(f ; x, y)$ we define the face function $f_{\Delta}$ by

$$
f_{\Delta}(x, y)=\sum_{(\alpha, \beta) \in \Delta} c_{\alpha, \beta} x^{\alpha} y^{\beta}
$$

In the space $\boldsymbol{R}^{2}$ where the Newton polygon $\Gamma_{+}(f ; x, y)$ is contained, we call $(u, v)$ the coordinates. For any linear form $P$ defined by $P(u, v)=a u+b v$, where $(a, b)$ is a pair of positive relatively prime integers, we define $d(P ; f)$ to be the smallest value of the restriction of $P$ to the Newton polygon $\Gamma_{+}(f ; x, y)$ and $\Delta(P ; f)$ be the face where this smallest value is taken. For simplicity we shall write $P={ }^{t}(a, b)$ and we simply denote $f_{P}$ instead of $f_{\Delta(P ; f)}$. Thus $f_{P}$ is a weighted homogeneous polynomial of weight ${ }^{t}(a, b)$ and degree $d(P ; f)$. For each face $\Delta$ of dimension 1 there is a unique linear form $P$ defined by the weight vector ${ }^{t}(a, b)$ of positive relatively prime integers such that $f_{P}=f_{\Delta}$ and furthermore we have a factorization of $f_{\Delta}$ :

$$
f_{\Delta}(x, y)=c x^{r} y^{s} \prod_{i=1}^{k}\left(y^{a}-\gamma_{\imath} x^{b}\right)^{\nu_{i}}
$$

with $d(P ; f)=\left(\sum_{\imath} \nu_{i}\right) a b+a r+b s$. We shall call $P={ }^{t}(a, b)$ the weight vector of the face $\Delta$.

There is a finite number of faces of dimension 1 in the boundary of the Newton polygon (notice that two of these faces are non-compact and parallel to the coordinate axes). We order the compact faces $\Delta_{2}(1 \leqq i \leqq m)$ of weights $P_{i}={ }^{t}\left(a_{\imath}, b_{i}\right)$ by the slopes $-a_{i} / b_{i}$ of the lines which contain them. We index such that $-\infty<-a_{1} / b_{1}<\cdots<-a_{m} / b_{m}<0$. The case $m=0$ means that the Newton boundary has one point, which means that $f(x, y)=x^{r} y^{s} u(x, y)$ where $u(x, y)$ is a unit at $O$.

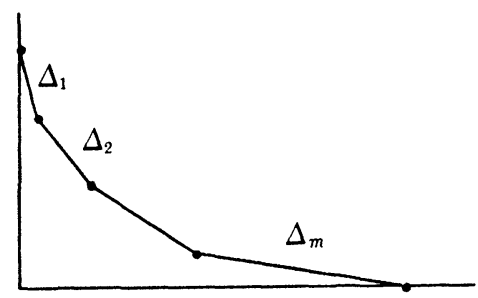

Figure (1.A) $(m=4)$ 
In the space $N^{+}$of positive dual vectors, we introduce an equivalence relation $\sim$ defined by $P \sim Q$ if and only if $\Delta(P ; f)=\Delta(Q ; f)$. The equivalence classes of this equivalence relation define a conical subdivision of $N^{+}$. By taking a line section which does not pass through the origin and intersects $N^{+}$(e.g. the line $x+y=1$ ), we obtain a simplex with a simplicial decomposition (in this case, a segment with a subdivision). We represent this simplex by the segment $\left[R_{-\infty}, R_{0}\right]$ with the subdivision given by the vertices $R_{-\infty}, P_{1}, \cdots, P_{m}, R_{0}$. We call this graph the dual Newton diagram $\Gamma^{*}(f ; x, y)$ of $f$ with respect to the coordinates $x$ and $y$. In this graph, the vertices correspond to the faces of the Newton polygon and their names correspond to the unique element in their equivalence class which is a primitive integral vector. So $R_{-\infty}={ }^{t}(1,0), P_{\imath}=$ ${ }^{t}\left(a_{\imath}, b_{i}\right)(1 \leqq i \leqq m)$ and $R_{0}=^{t}(0,1)$. The edges correspond to the vertices of the Newton boundary. Because of its particular shape, this graph is called a bamboo, i. e. a tree without branches, linking $R_{-\infty}$ and $R_{0}$ through $P_{1}, \cdots, P_{m}$. Let

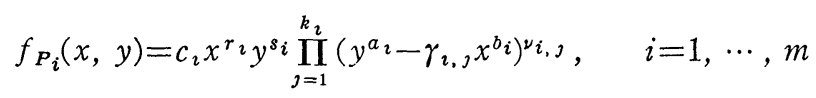

Definition (1.1). We say that the coordinate $x$ is bad for $f$ at the point $O$ if the first face function $f_{P_{1}}$ satisfies one of the following conditions.

(1) $m \geqq 1$ and $f_{P_{1}}(x, y)$ is of the form $c_{1} y^{s_{1}}\left(y^{a_{1}}-\gamma_{1,1} x\right)^{\nu_{1,1}}$ i. e., $r_{1}=0, P_{1}=$ ${ }^{t}\left(a_{1}, 1\right)$ and $k_{1}=1$.

(2) $\quad m=1$ and $f_{P_{1}}(x, y)=c_{1}\left(y-\gamma_{1,1} x\right)^{\nu_{1,1}}\left(y-\gamma_{1,2} x\right)^{\nu_{1,2}}$ i. e., $r_{1}=s_{1}=0, P_{1}=t(1,1)$ and $k_{1}=2$.

Similarly $y$ is called bad if the last face function $f_{P_{m}}(x, y)$ satisfies either (1)' or (2) where

$(1)^{\prime} \quad m \geqq 1$ and $f_{P_{m}}(x, y)=c_{m} x^{r_{m}}\left(y-\gamma_{m, 1} x^{b_{m}}\right)^{\nu_{m, 1}}$ i.e., $s_{m}=0, \quad P_{m}={ }^{t}\left(1, b_{m}\right)$ and $k_{m}=1$.

We say that the coordinate $x$ (resp. $y$ ) is quasi-good for $f$ at the point $O$ if it is not bad. We say that a coordinate system $(x, y)$ is quasi-good for $f$ at the point $O$ if both $x$ and $y$ are quasi-good for $f$. Notice that if $m=0$, the coodinates $x$ and $y$ are always quasi-good. In case (2), both $x$ and $y$ are bad coodinates.

We will see later that there exists a toric bowing-up $\pi: X \rightarrow C^{2}$ which is canonically associated with the dual Newton diagram $\Gamma^{*}(f ; x, y)$ (see (2.6)). The corresponding dual graph of the exceptional divisors is a bamboo. Then $x$ is a bad coordinate if and only if the divisor $\hat{E}\left(P_{1}\right)$ which corresponds to the weight vector $P_{1}$ intersects at most two irreducible components of the divisor $(\pi * f)$ (see Lemma $(2.12)$ ).

Remark. In the case that the function $f$ is analytically irreducible at $O$, the Newton boundary has only one face and it touches to the both axis and the coordinate $x$ (respectively $y$ ) is bad for $f$ if and only if the slope of the Newton boundary (resp. the inverse of the slope of the Newton boundary) is an integer. 
DEFINITION (1.2). We say that $x$ is good if $x$ is quasi-good and one of the following condition is satisfied.

(i) $m=0$ or (ii) $m \geqq 1$ and $b_{1}>1$ or

(iii) $m \geqq 1, b_{1}=1$,

$$
f_{P_{1}}(x, y)=c_{1} x^{r_{1}} y^{s_{1}} \prod_{\jmath=1}^{k_{1}}\left(y^{a_{1}}-\gamma_{1, j} x\right)^{\nu_{1,},} \quad\left(b_{1}=1\right)
$$

and for any change of coordinates $x^{\prime}=x-\gamma_{1, j}^{-1} y^{a_{1}}+h(y), y^{\prime}=y$ with $1 \leqq \jmath \leqq k_{1}$ and val $h>a_{1}$ such that $x^{\prime}$ is a quasi-good for $f^{\prime}\left(x^{\prime}, y^{\prime}\right):=f\left(x^{\prime}+\gamma_{1, j}^{-1} y^{\prime a_{1}}-h\left(y^{\prime}\right), y^{\prime}\right)$, the number of compact faces of $\Gamma\left(f^{\prime} ; x^{\prime}, y^{\prime}\right)$ is $m$.

Similarly we say that $y$ is good if $y$ is a quasi-good coordinate and (i) $m=0$ or (ii) $m \geqq 1$ and $a_{m}>1$ or (iii) $m \geqq 1, a_{m}=1$ and

$$
f_{P_{m}}(x, y)=c_{m} x^{r_{m}} y^{s_{m}} \prod_{\jmath=1}^{k}\left(y-\gamma_{m, \jmath} x^{b_{m}}\right)^{\nu_{1, \jmath}} \quad\left(a_{m}=1\right)
$$

and for any change of coordinates $x^{\prime}=x, y^{\prime}=y-\gamma_{m, j} x^{b} m+g(x)$ with $1 \leqq j \leqq k_{m}$ and val $g>b_{m}$ such that $y^{\prime}$ is a quasi-good for $f^{\prime}\left(x^{\prime}, y^{\prime}\right):=f\left(x^{\prime}, y^{\prime}+\right.$ $\left.\gamma_{m, j} x^{\prime b m}-g\left(x^{\prime}\right)\right)$, the number of compact faces of $\Gamma\left(f^{\prime} ; x^{\prime}, y^{\prime}\right)$ is $m$.

If both $x$ and $y$ are good, we say that $(x, y)$ is a good system of coordinates.

As the coordinate change of type (iii) in definition (1.2) does not change the other part of the Newton boundary $\Gamma(f ; x, y) \cap\left\{u \geqq r_{1}\right\}=\cup_{\jmath=2}^{m} \Delta_{\jmath}$, the condition (iii) implies that the face $\Delta_{1}$ does not split into more than two faces.

We will see later that $x$ is a good coordinate for $f(x, y)$ if and only if $\left(r_{1}, k_{1}\right) \neq(0,1)$ and for each $\jmath=1, \cdots, k_{1}$ there exists analytic functions $h_{j}(y)$ with val $h_{j}>a_{1}$ such that $\left(x-\gamma_{1, j}^{-1} y^{a_{1}}+h_{j}(y)\right)^{\nu_{1, j}}$ divides $f(x, y)$ (see Sublemma (1.9)). Geometrically this implies that the function $\pi * f$ has only normal crossing singularities on the exceptional divisor $\hat{E}\left(P_{1}\right)$ (Lemma (2.9)).

Example (1.2.1). We give two basic examples.

(A) Let us consider the case

$$
f(x, y)=\left(x+y^{2}\right)^{2}+x^{3} y
$$

Then the Newton boundary has only one face and the corresponding face function is

$$
f_{P_{1}}(x, y)=\left(x+y^{2}\right)^{2}
$$

As is easy to see, $x$ is not even a quasi-good coordinate for $f$. We will see later that $C$ is irreducible but the strict transform of $C$ on the total space $X$ of the toric blowing-up has a cusp singularity at the intersection with the exceptional divisor. To give an example of a curve $C=\{k(x, y)=0\}$ for which $x$ is a quasi-good but not good coordinate, we can simply take $k(x, y)=f(x, y)$. $h(x, y)$ where $\Gamma(h ; x, y)$ does not have any face whose slope is equal or sharper than -2 . Namely we assume that 


$$
h_{P}(x, y)=x^{a} y^{b} \quad \text { for any } P={ }^{t}(p, q), p / q \leqq-2
$$

As an easiest example, we can take $h(x, y)=x$ and let

$$
k(x, y)=x\left(x+y^{2}\right)^{2}+x^{4} y
$$

Then the Newton boundary has only one face and the corresponding face function is

$$
k_{P_{1}}(x, y)=x\left(x+y^{2}\right)^{2}
$$

Thus it is easy to see that $x, y$ are quasi-good coordinates for $k$. However $x$ is not a good coordinate. In fact, take the change of coordinate:

$$
x^{\prime}=x+y^{2}, \quad y^{\prime}=y
$$

Then in this coordinate, $f$ is written as

$$
k^{\prime}\left(x^{\prime}, y^{\prime}\right)=\left(x^{\prime}-y^{\prime 2}\right) x^{\prime 2}+\left(x^{\prime}-y^{\prime 2}\right)^{4} y^{\prime}
$$

Now $\Gamma\left(k^{\prime} ; x^{\prime}, y^{\prime}\right)$ has two compact faces and the face function of the first face is

$$
k_{P_{1}^{\prime}}^{\prime}\left(x^{\prime}, y^{\prime}\right)=y^{\prime 2}\left(-x^{\prime 2}+y^{\prime 7}\right)
$$

Thus it is easy to see that $x^{\prime}$ is a quasi-good (in fact a good) coordinate. Thus $x$ is not a good coordinate by the definition. For the geometrical interpretation, see Example (2.10.1).
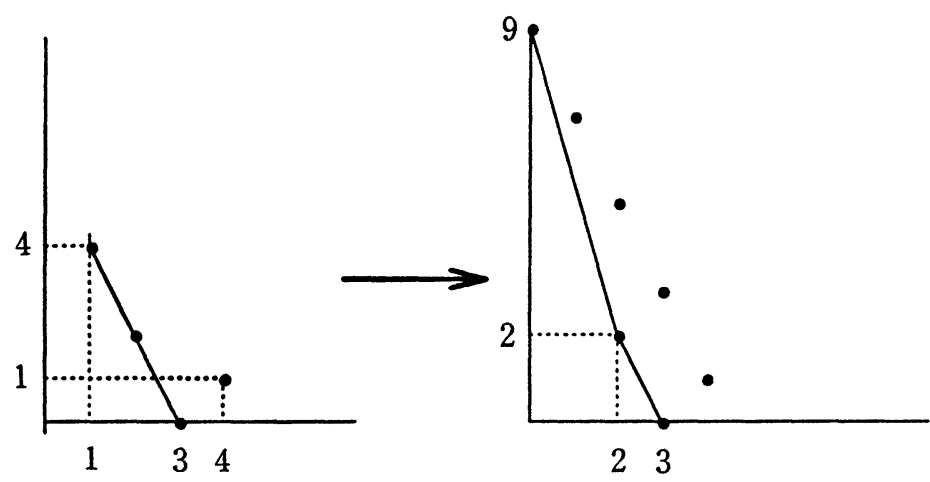

Figure (1.2.1.A)

(B) Let us consider the function

$$
g(x, y)=\left(x+y^{2}\right)\left(x-y^{2}\right)\left(x-y^{2}+y^{2+m}\right)
$$

The Newton boundary has only one face and the curve $g(x, y)=0$ has three irreducible components. Note that 


$$
g_{P_{1}}(x, y)=\left(x+y^{2}\right)\left(x-y^{2}\right)^{2}
$$

It is obvious that $x$ is a quasi-good coordinate for $g$. We assert that $x$ is not a good coordinate. In fact, let

$$
x^{\prime}=x-y^{2}, \quad y^{\prime}=y
$$

and let $g^{\prime}\left(x^{\prime}, y^{\prime}\right):=g\left(x^{\prime}+y^{\prime 2}, y^{\prime}\right)$. Then $\Gamma\left(g^{\prime} ; x^{\prime}, y^{\prime}\right)$ has two faces and the first face has the weight vector $P^{\prime}={ }^{t}(2+m, 1)$ and

$$
\begin{aligned}
& g^{\prime}\left(x^{\prime}, y^{\prime}\right)=x^{\prime}\left(x^{\prime}+2 y^{\prime 2}\right)\left(x^{\prime}+y^{\prime 2+m}\right) \\
& g_{P^{\prime}}^{\prime}\left(x^{\prime}, y^{\prime}\right)=2 x^{\prime} y^{\prime 2}\left(x^{\prime}+y^{\prime 2+m}\right) \\
& g_{P_{1}}^{\prime}\left(x^{\prime}, y^{\prime}\right)=x^{\prime 2}\left(x^{\prime}+2 y^{\prime 2}\right)
\end{aligned}
$$

$x^{\prime}$ is obviously a quasi-good coordinate for $G$. In fact, it is also a good coordinate for $f^{\prime}$ by Sublemma (1.9) as $x^{\prime}+y^{\prime 2+m}$ divides $g^{\prime}\left(x^{\prime}, y^{\prime}\right)$. Let $\pi: X \rightarrow C^{2}$ be the canonical toric blowing-up with respect to $(x, y)$. Then the strict transforms of the irreducible components $x-y^{2}=0$ and $x-y^{2}+y^{2+m}=0$ are smooth but they intersects with intersection multiplicity $m$ at the point where they intersect transversely with $\hat{E}\left(P_{1}\right)$.

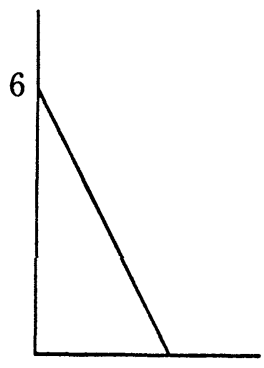

3

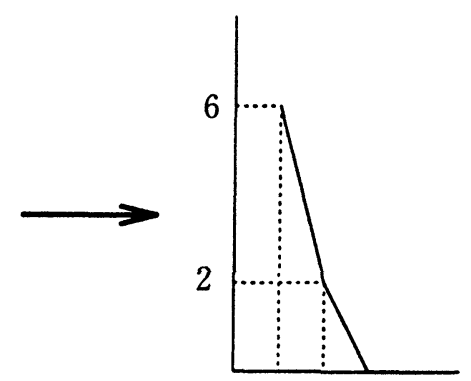

$\begin{array}{lll}1 & 2 & 3\end{array}$

Figure (1.2.1.B.)

See also Example (2.10.1). Let $h(x, y)$ be as in (A). The same assertion is true for

$$
k(x, y)=\left(x+y^{2}\right)\left(x-y^{2}\right)\left(x-y^{2}+y^{2+m}\right) h(x, y)
$$

We have the following fundamental lemma:

LEMMA (1.3). Assume that $x$ is a bad coordinate for a given $f(x, y)$ as above and assume that

$$
f_{P_{1}}(x, y)=c_{1} y^{s_{1}}\left(y^{a_{1}}-\gamma_{1,1} x\right)^{\nu_{1,1}}
$$

Then there exists an analytic change of coordinates $\left(x^{\prime}, y^{\prime}\right)$ : 


$$
x=x^{\prime}+\gamma_{1,1}^{-1} y^{\prime a_{1}}+h\left(y^{\prime}\right), \quad y=y^{\prime}
$$

where $h\left(y^{\prime}\right)$ is a germ of an analytic function of $y^{\prime}$ with val $h>a_{1}$ so that $x^{\prime}$ is a quasi-good coordinate for $f^{\prime}\left(x^{\prime}, y^{\prime}\right):=f\left(x^{\prime}+\gamma_{1,1}^{-1} y^{\prime a_{1}}+h\left(y^{\prime}\right), y^{\prime}\right)$. Here val is the valuation of the corresponding series. Furthermore if $y$ is quasi-good for $f(x, y), y^{\prime}$ is also quasi-good for $f^{\prime}\left(x^{\prime}, y^{\prime}\right)$.

The similar assertion is true for the coordinate $y$ if $y$ is a bad coordinate.

COROLLARY (1.4). For any given $f(x, y)$, there exists an analytic change of coordinates

$$
x=\varphi\left(x_{1}, y_{1}\right) \quad y=\phi\left(x_{1}, y_{1}\right)
$$

where $\left(x_{1}, y_{1}\right)$ is a quasi-good coordinate system for $f_{1}\left(x_{1}, y_{1}\right)=f\left(\varphi\left(x_{1}, y_{1}\right), \phi\left(x_{1}, y_{1}\right)\right)$. Furthermore if $x$ (resp. $y$ ) is already quasi-good we can choose this change of coordinates such that $x=x_{1}$ (resp. $y=y_{1}$ ).

Proof of Corollary (1.4). Assume that $x$ is a bad coordinates. If $x$ is as in Lemma (1.3), we apply Lemma (1.3). If $x$ is bad and the condition (2) is satisfied in Definition (1.1). Namely assume that $m=1$ and $f_{P_{1}}(x, y)=$ $c_{1}\left(y-\gamma_{1,1} x\right)^{\nu_{1,1}}\left(y-\gamma_{1,2} x\right)^{\nu_{1,2}}$. Then we first take the coordinate change

$$
x^{\prime}=y-\gamma_{1,1} x, \quad y^{\prime}=y-\gamma_{1,2} x
$$

If either $x^{\prime}$ or $y^{\prime}$ is still a bad coordinate, we come to a situation as in Lemma (1.3). If $x^{\prime}$ or $y^{\prime}$ is bad, we apply Lemma (1.3) (twice if necessary). This proves the assertion.

Proof of Lemma (1.3). Suppose that $x$ is a bad coordinate and we may assume

$$
f_{P_{1}}(x, y)=c y^{s}\left(y^{a}-\gamma x\right)^{\nu}
$$

for convenience. Note $(\nu, s) \in \Gamma(f ; x, y)$ be the first interior vertex of the Newton boundary $\Gamma(f ; x, y)$. (The first interior vertex of the Newton boundary which has the minimal positive $u$-coordinate.) We first consider the change of coordinates:

$$
x=x^{\prime}+\gamma^{-1} y^{\prime a}+h\left(y^{\prime}\right), \quad y=y^{\prime}
$$

Here $h\left(y^{\prime}\right)$ is any germ of an analytic function of $y^{\prime}$ with val $h>a$, where val is the valuation of the corresponding series. Let

$$
f^{\prime}\left(x^{\prime}, y^{\prime}\right):=f\left(x^{\prime}+\gamma^{-1} y^{\prime a}+h\left(y^{\prime}\right), y^{\prime}\right)
$$

ASSERTION (1.5). The intersection $\Gamma\left(f^{\prime} ; x^{\prime}, y^{\prime}\right) \cap\{u \geqq \nu\}$ equals to $\Gamma(f ; x, y)$ $\cap\{u \geqq \nu\}$ and $f_{\Delta_{i}}^{\prime}\left(x^{\prime}, y^{\prime}\right)=f_{\Delta_{i}}(x, y)$ for any $i=2, \cdots, m$. In $\Gamma\left(f^{\prime} ; x^{\prime}, y^{\prime}\right) \cap\{u \leqq \nu\}$, either there are no faces or any new face is sharper than $\Delta_{1}$. Let $\left(\nu^{\prime}, s^{\prime}\right) \in$ $\Gamma\left(f^{\prime} ; x^{\prime}, y^{\prime}\right)$ be the first interior vertex. Then $\nu^{\prime} \leqq \nu$. If $y$ is quasi-good for $f, y^{\prime}$ is also quasi-good for $f^{\prime}$. 
Proof. Let $P={ }^{t}(p, q) \in N^{+}$be a dual vector. Then we have

$$
\begin{aligned}
f_{P}^{\prime}\left(x^{\prime}, y^{\prime}\right) & =\left(f\left(x^{\prime}+\gamma^{-1} y^{\prime a}+h\left(y^{\prime}\right), y^{\prime}\right)\right)_{P} \\
& = \begin{cases}f_{P}\left(x^{\prime}, y^{\prime}\right) & \text { if } p / q<a \\
f_{P}\left(x^{\prime}+\gamma^{-1} y^{\prime a}, y^{\prime}\right)=c y^{\prime s} x^{\prime \nu} & \text { if } p / q=a\end{cases}
\end{aligned}
$$

Thus, for any dual vector $P={ }^{t}(p, q)$ such that $p / q<a$, we have $\Delta(P ; f)=\Delta\left(P ; f^{\prime}\right)$ and $f_{P}^{\prime}\left(x^{\prime}, y^{\prime}\right)=f_{P}(x, y)$. This proves the first assertion. Assume that $y$ is quasi-good for $f$. Then we have to show that $y^{\prime}$ is also quasi-good. If the number of faces in the Newton boundary $m$ is greater than $1, y^{\prime}$ is obviously quasi-good for $f^{\prime}$ by the above argument. Assume that $m=1$. Then $f_{P_{1}}(x, y)$ $=c y^{s}\left(y^{a}+\gamma x\right)^{\nu}$. In this case $y$ is bad if and only if $s=0$ and $a=1$. If $y$ is quasi-good and $s>0$, it is easy to see that $y^{\prime s}$ divides $f^{\prime}$, so $y^{\prime}$ is also quasigood for $f^{\prime}$. If $y$ is quasi-good and $s=0$, we must have $a>1$. By the above argument, $\Gamma\left(f^{\prime}\right)$ does not have any face with the corresponding covector $P=$ ${ }^{t}(p, q)$ with $p / q \leqq 1$ and $y^{\prime}$ is quasi-good for $f^{\prime}$. Thus in any case, the coordinate function $y^{\prime}$ is quasi-good for $f^{\prime}$. This proves the above assertion. Q.E.D.

Now we use the preceding assertion to prove Lemma (1.3). Let $g$ and $h$ be germs of analytic functions at the origin such that the ideal $(g, h)$ generated by $g$ and $h$ has a finite codimension in the ring of germs of analytic functions 0 . Recall that the intersection multiplicity $m(f, g ; O)$ of $\{g=0\}$ and $\{h=0\}$ at the origin is defined by $\operatorname{dim} \theta /(f, g)$.

Let

$$
\mathcal{S}=\left\{g \in \mathcal{O} ; g(x, y)=x-\gamma^{-1} y^{a}-h(y), \text { val } h>a\right\}
$$

In the above notation, we have:

$$
\begin{aligned}
m(f, x ; O)=\operatorname{dim} \theta /(f, x) & =\operatorname{val} f(0, y)=s+a \nu=d\left(P_{1} ; f\right) \\
m\left(f, x-\gamma^{-1} y^{a}-h(y) ; O\right) & =\operatorname{dim} \theta /\left(f, x-\gamma^{-1} y^{a}-h(y)\right) \\
& =\operatorname{val} f\left(\gamma^{-1} y^{a}+h(y), y\right) \\
& >s+a \nu .
\end{aligned}
$$

Thus we have

$$
m(f, g ; O)>m(f, x ; O) \quad \text { for any } g \in \mathcal{S} .
$$

We consider the change of coordinates:

and let

$$
x_{1}=x-\gamma^{-1} y^{a} \quad y_{1}=y
$$

$$
f_{1}\left(x_{1}, y_{1}\right):=f\left(x_{1}+\gamma^{-1} y_{1}^{a}, y_{1}\right)
$$

Call this change of coordinates the canonical change of coordinates. If $x_{1}$ is not 
quasi-good for $f_{1}$, we take the canonical change of coordinates $\left(x_{2}, y_{2}\right)$ and we continue this operations. Note that a finite composition of canonical changes of coordinates is a change of coordinates of the form:

$$
x=x^{\prime}+\gamma^{-1} y^{\prime a}+h\left(y^{\prime}\right) \quad y=y^{\prime}
$$

as we have used in the above assertion. Thus we have two possibilities.

(a) There exists a $j_{0}$ such that $x_{\jmath_{0}}$ is a quasi-good coordinate for $f_{\jmath_{0}}\left(x_{\jmath_{0}}, y_{\jmath_{0}}\right)$.

(b) For any $j>0, x_{j}$ is not quasi-good for $f_{j}$.

In the case of (a), we can simply take $x^{\prime}=x_{\jmath_{0}}, y^{\prime}=y_{\jmath_{0}}$. Note that $h(y)$ is a polynomial in this case. In the case of (b), the first vertex of the Newton boundary which we denote by $\left(\nu_{j}, s_{j}\right)$ does not change after some $\jmath_{0}>0$, so we may assume that $\nu_{j}=\nu_{j+1}$ and $m\left(f, x_{j+1}\right)>m\left(f, x_{j}\right)$ for any $\jmath$. In particular, this implies that $\sup _{g \subseteq s} m(f, g)=\infty$. So, we assert

ASSERTION (1.6). Assume that $\sup _{g \in S} m(f, g)=\infty$. Then there is a $g(x, y)$ $\in \mathcal{S}$ such that $g \mid f$.

Assuming this assertion, we take $x^{\prime}=g(x, y)$ and $y^{\prime}=y$ to complete the proof.

To prove the last assertion, we consider the intersection multlplicity. Let $f=k_{1}^{r_{1}} \ldots k_{s}^{r_{s}}$ be the factorization in the ring $\mathcal{O}$ of $f$ into irreducible functions. First by the linearity of the intersection multiplicity, $m(f, g)=\sum_{\imath=1}^{s} r_{i} m\left(k_{\imath}, g\right)$. So if $\sup _{g \in S} m(f, g)=\infty$, there is $i, 1 \leqq i \leqq s$, such that $\sup _{g \in S} m\left(k_{\imath}, g\right)=\infty$. Then using the Puiseux expansion of $k_{\imath}$ and the equality

$$
m\left(k_{\imath} ; x+\gamma^{-1} y^{a}+h(y)\right)=\operatorname{val} k_{\imath}\left(-\gamma^{-1} y^{a}-h(y), y\right),
$$

it is easy to see that $\sup _{g \in S} m\left(k_{\imath}, g\right)=\infty$ if and only if in the complete ring $\hat{\mathcal{O}}$ of formal power series, there is an element $g$ of $\mathcal{S}$ (which is necessarily irreducible in $\mathcal{O}$ ) which divides $k_{\imath}$. But being irreducible in $\mathcal{O}, k_{\imath}$ is irreducible in its completion $\hat{\mathcal{O}}$. Therefore $\left\{k_{\imath}=0\right\}$ is non-singular and $\xi k_{i} \in \mathcal{S}$ for some unit $\xi$ in $\mathcal{O}$. Namely there exists a $g \in \mathcal{S}$ such that $g$ divides $f$. Q.E.D.

This completes the proof of Lemma (1.3).

Now we extend Lemma (1.3) for good coordinates.

LEMMA (1.7). Assume that $x$ is a quasi-good coordinate which is not good and assume that $f_{P_{1}}(x, y)=c_{1} x^{r_{1}} y^{s_{1}} \prod_{\jmath=1}^{k_{1}}\left(y^{a_{1}}-\gamma_{1, j} x\right)^{\nu_{1, \jmath}}$. Then there exists $a j$ $\left(1 \leqq j \leqq k_{1}\right)$ and a coordinate change $\left(x^{\prime}, y^{\prime}\right)$ :

$$
x=x^{\prime}+\gamma_{1,3}^{-1} y^{\prime a_{1}}+h\left(y^{\prime}\right), \quad y=y^{\prime}
$$

with val $h>a_{1}$ and $x^{\prime}$ is good for $f^{\prime}\left(x^{\prime} y^{\prime}\right):=f\left(x^{\prime}+\gamma_{1, j}^{-1} y^{\prime a_{1}}+h\left(y^{\prime}\right), y^{\prime}\right)$. If $y$ is good, $y^{\prime}$ is also good. A similar assertion is true for $y$ coodinates. 
COROLLARY (1.8). For any given $f(x, y)$, there exists an analytic change of coordinates

$$
x=\varphi\left(x_{1}, y_{1}\right) \quad y=\psi\left(x_{1}, y_{1}\right)
$$

where $\left(x_{1}, y_{1}\right)$ is a good coordinate system for $f_{1}\left(x_{1}, y_{1}\right)=f\left(\varphi\left(x_{1}, y_{1}\right), \psi\left(x_{1}, y_{1}\right)\right)$. Furthermore if $x$ (resp. $y$ ) is already good we can choose this change of coordinates such that $x=x_{1}$ (resp. $\left.y=y_{1}\right)$

To prove the assertion for the good coordinates, we first prove the following criterion for the goodness.

Sublemma (1.9). Let $f_{P_{1}}(x, y)=c_{1} x^{r_{1}} y^{s_{1}} \prod_{j=1}^{k_{1}}\left(y^{a_{1}}-\gamma_{1, j} x\right)^{\nu_{1}, \jmath}$. The coordinate $x$ is good if and only if $\left(r_{1}, k_{1}\right) \neq(0,1)$ and for each $j=1, \cdots, k_{1}$ there exists analytic functions $h_{j}(y)$ with val $h_{j}>a_{1}$ such that $\left(x-\gamma_{1, j}^{-1} y^{a_{1}}+h_{j}(y)\right)^{\nu_{1,},}$ divides $f(x, y)$.

Sublemma (1.9) and Lemma (2.9) give a geometrical interpretation for the good coordinate.

Proof. Assume that $x$ is good. Let $x^{\prime}=x-\gamma_{1, j}^{-1} y^{a_{1}}, y^{\prime}=y$ and let $f^{\prime}\left(x^{\prime}, y^{\prime}\right)$ : $=f\left(x^{\prime}+\gamma_{1, j} y^{a_{1}}, y^{\prime}\right)$. As in the proof of Assertion (1.6), we can see easily that

$$
\Gamma\left(f^{\prime} ; x^{\prime}, y^{\prime}\right) \cap\left\{u \geqq r_{1}+\sum_{j=1}^{k_{1}} \nu_{1, j}\right\}=\Gamma(f ; x, y) \cap\left\{u \geqq r_{1}+\sum_{j=1}^{k_{1}} \nu_{1, j}\right\} .
$$

Obviously $f_{P_{1}}(x, y)$ changes as

$$
f_{P_{1}}^{\prime}\left(x^{\prime}, y^{\prime}\right)=\left(x^{\prime}+\gamma_{1, j}^{-1}, y^{\prime a_{1}}\right)^{r_{1}} y^{s_{1}} x^{\prime \nu_{1, j}} \prod_{l \neq j}\left(\left(1-\gamma_{1, l} \gamma_{1, j}^{-1}\right) y^{\prime a_{1}}-\gamma_{1, l} x^{\prime}\right)^{\nu_{1, l}}
$$

As $x$ is assumed to be good, $r_{1}>0$ if $k_{1}=1$. Thus $f_{P}^{\prime}$ is not a monomial. Let $\Delta_{1}^{\prime}$ be the support of this polynomial. If $\Gamma\left(f^{\prime} ; x^{\prime}, y^{\prime}\right) \cap\left\{u<\nu_{1, j}\right\}=\emptyset$, this implies that $x^{\prime \nu_{1, j}} \mid f^{\prime}$ and the assertion is proved. Assume that $\Gamma\left(f^{\prime} ; x^{\prime}, y^{\prime}\right) \cap\left\{u \leqq \nu_{1, j}\right\}$ contains faces. Let them $\Delta_{1,1}^{\prime}, \cdots, \Delta_{1, t}^{\prime}$. Thus $\Delta_{1,1}^{\prime}, \cdots, \Delta_{1, t}^{\prime}, \Delta_{1}^{\prime}$ and $\Delta_{2}, \cdots, \Delta_{m}$ is the faces of $\Gamma\left(f^{\prime} ; x^{\prime}, y^{\prime}\right)$. By the goodness of $x, x^{\prime}$ is necessarily not quasigood. Thus we can write

$$
f_{\Delta_{1,1}^{\prime}}^{\prime}\left(x^{\prime}, y^{\prime}\right)=c^{\prime} y^{\prime s^{\prime}}\left(y^{a}-\gamma^{\prime} x^{\prime}\right)^{\nu^{\prime}} .
$$

Note that $a^{\prime}>a_{1}$. Using Lemma (1.3), we can take a coordinate change

$$
x^{\prime \prime}=x^{\prime}-\gamma^{\prime-1} y^{\prime a}+h\left(y^{\prime}\right), \quad y^{\prime \prime}=y^{\prime}
$$

so that $x^{\prime \prime}$ is a quasi-good coordinate for $f^{\prime \prime}\left(x^{\prime \prime}, y^{\prime \prime}\right):=f^{\prime}\left(x^{\prime \prime}+\gamma^{\prime-1} y^{\prime \prime} a_{1}-h\left(y^{\prime \prime}\right), y^{\prime \prime}\right)$ and the faces $\Delta_{1,2}^{\prime}, \cdots, \Delta_{1, t}^{\prime}, \Delta_{1}^{\prime}, \Delta_{2}, \cdots, \Delta_{m}$ are unchanged under this change of coordinates. Again by the goodness of $x$, this is possible if and only if $t=1$ and $\Gamma\left(f^{\prime \prime} ; x^{\prime \prime}, y^{\prime \prime}\right) \cap\left\{u<\nu_{1, j}\right\}=\emptyset$. This implies that $x^{\prime \prime \nu_{1, j}} \mid f$. As we can write $x^{\prime \prime}=x-\gamma_{1, j}^{-1} y^{a_{1}}+h^{\prime}(y), y^{\prime \prime}=y$ with val $h^{\prime}>a_{1}$, the necessity is proved. 
Now we consider the sufficiency. Assume that for each $\jmath=1, \cdots, k_{1}$, there exists some $h_{3}$ with val $h_{j}>a_{1}$ such that $\left(x-\gamma_{1, j}^{-1} y^{a_{1}}+h_{j}(y)\right)^{\nu_{1}, j}$ divides $f$. Let $X=x-\gamma_{1, j}^{-1} y^{a_{1}}+h_{j}(y), Y=y$ and let $F(X, Y):=f\left(X+\gamma_{1, j}^{-1} Y^{a_{1}}-h_{j}(Y), Y\right)$. The assumption implies that $\Gamma(F ; X, Y) \cap\left\{u<\nu_{1, j}\right\}=\emptyset$. Note that the Newton boundary $\Gamma(F ; X, Y)$ is same with $\Gamma(f ; x, y)$ for $u \geqq r_{1}+\sum_{j=1}^{k_{1}} \nu_{1, j}$ and

$$
F_{P_{1}}(X, Y)=c_{1}\left(X+\gamma_{1, j}^{-1} Y^{a_{1}}\right)^{r_{1}} Y^{s_{1}} X^{\nu_{1, \nu}} \prod_{l \neq j}\left(\left(1-\gamma_{1, l} \gamma_{1, j}^{-1}\right) Y^{a_{1}}-\gamma_{1, l} X\right)^{\nu_{1, l}}
$$

Now let $x^{\prime}=x-\gamma_{1, j}^{-1} y^{a_{1}}+\phi(y), y^{\prime}=y$ be an arbitrary change of coordinates with val $\phi>a_{1}$. Then the following assertion shows that $x$ is a good coordinate for $f$.

ASSERTION (1.9.1). Assume that $x^{\prime}$ is a quasi-good coordinate for

$$
f^{\prime}\left(x^{\prime}, y^{\prime}\right):=f\left(x^{\prime}+\gamma_{1, j}^{-1} y^{\prime a_{1}}-\phi\left(y^{\prime}\right), y^{\prime}\right)
$$

Then $\phi\left(y^{\prime}\right)=h_{j}\left(y^{\prime}\right)$ and $x^{\prime}=X$.

Proof. It is easy to see that $x^{\prime}=X-h_{j}(Y)+\phi(Y), y^{\prime}=Y$. Thus we have

$$
f^{\prime}\left(x^{\prime}, y^{\prime}\right)=F\left(x^{\prime}-h_{j}\left(y^{\prime}\right)+\phi\left(y^{\prime}\right), y^{\prime}\right)
$$

Let $\phi\left(y^{\prime}\right):=\phi\left(y^{\prime}\right)-h_{j}\left(y^{\prime}\right)$. Assume that $\phi \neq 0$ and let

$$
\phi\left(y^{\prime}\right)=\gamma^{\prime} y^{\prime a^{\prime}}+(\text { higher terms) }
$$

and let $P={ }^{t}\left(a^{\prime}, 1\right)$. Note that $a^{\prime}>a_{1}$. Thus it is easy to see that

$$
f_{P}^{\prime}\left(x^{\prime}, y^{\prime}\right)=\left(F_{P_{1}}\left(x^{\prime}+\gamma^{\prime} y^{a^{\prime}}, y^{\prime}\right)\right)_{P}=c_{1}^{\prime}\left(x^{\prime}+\gamma^{\prime} y^{a^{\prime}}\right)^{\nu_{1,}, y^{\prime \xi}}
$$

with $\xi=s_{1}+a_{1}\left(r_{1}+\sum_{l \neq j} \nu_{1, l}\right)$. Thus $x^{\prime}$ is not quasi-good which is a contradiction. Therefore if $x^{\prime}$ is quasi-good for $f^{\prime}$, then $x^{\prime}=X$. Q.E.D.

Proof of Lemma (1.7). Assume first that $x$ is quasi-good and let

$$
f_{P_{1}}(x, y)=c_{1} x^{r_{1}} y^{s_{1}} \prod_{\jmath=1}^{k_{1}}\left(y^{a_{1}}-\gamma_{1, j} x\right)^{\nu_{1, \jmath}}
$$

Assume that $x$ is not good. By the definition, there exists a coordinate change

$$
x^{\prime}=x-\gamma_{1, y}^{-1} y^{a_{1}}+\phi(y), \quad y^{\prime}=y
$$

for some $j$ and $\phi(y)$ with val $\phi>a_{1}$ such that $x^{\prime}$ is quasi-good for

$$
f^{\prime}\left(x^{\prime}, y^{\prime}\right):=f\left(x^{\prime}+\gamma_{1, j}^{-1} y^{\prime a_{1}}-\phi\left(y^{\prime}\right), y^{\prime}\right)
$$

and in $\Gamma\left(f^{\prime} ; x^{\prime}, y^{\prime}\right) \Delta_{1}$ splits into more that two faces in $\Gamma\left(f^{\prime} ; x^{\prime}, y^{\prime}\right)$. If $x^{\prime}$ is not good, we continue this operation. Such an operation strictly increases the number of faces of the corresponding Newton boundary and only the first 
face $\Delta_{1}$ is changed into several faces. Note that the $u$ coordinate of the right end of the first face $\Delta_{1}$ is strictly decreasing under this operation. Thus this operation stops after finite steps. Namely we obtain a quasi-good coordinate system $\left(x_{1}, y_{1}\right)$ where $x_{1}$ is good. The composition of such changes of coordinates is again a quasi-good coordinate change which we are considering: $x_{1}=$ $x-\gamma_{1, j}^{-1} y^{a_{1}}+h(y), y_{1}=y$ with val $h>a_{1}$.

Now assume that $y$ is good from the beginning. We have to show that $y_{1}$ is also good. Let $f_{1}\left(x_{1}, y_{1}\right):=f\left(x_{1}+\gamma_{1, j}^{-1} y_{1}^{a_{1}}-h\left(y_{1}\right), y_{1}\right)$ and let

$$
f_{P_{m}}(x, y)=c_{m} x^{r_{m}} y^{s_{m}} \prod_{\jmath=1}^{k m}\left(y^{a} m-\gamma_{m, j} x^{b_{m}}\right)^{\nu_{m, \jmath}}
$$

Note that

$$
f_{1, P_{m}}\left(x_{1}, y_{1}\right)=\left\{\begin{array}{l}
c_{m} x_{1}^{r_{m} m} y_{1}^{s_{m} m} \prod_{j=1}^{k}{ }_{j}\left(y_{1}^{a} m-\gamma_{m, j} x_{1}^{b m}\right)^{\nu_{m} m, j}, \quad m>1 \\
c_{1}\left(x_{1}+\gamma_{1, j}^{-1} y_{1}^{a_{1}}\right)^{r_{1}} y_{1}^{s_{1}} x_{1}^{\nu_{1}, j} \prod_{l \neq j}\left(\left(1-\gamma_{1, l} \gamma_{1, j}^{-1}\right) y_{1}^{a_{1}}-\gamma_{1, l} x_{1}\right)^{\nu_{1}, l}, \quad m=1
\end{array}\right.
$$

If $a_{m}>1, y_{1}$ is good by the definition. Assume that $a_{m}=1$. By Sublemma (1.9), for each $1 \leqq l \leqq k_{m}$, there exists $h_{l}(x)$ with val $h_{l}>b_{m}$ such that $\left(y-\gamma_{m, l} x^{b_{m}}+\right.$ $\left.h_{l}(x)\right)^{\nu, l} \mid f(x, y)$. Let $x^{\prime \prime}=x, y^{\prime \prime}=y-\gamma_{m, l} x^{b_{m}}+h_{l}(x)$. As $x_{1}=x-\gamma_{1, j} y^{a_{1}}+h(y)$ and $y_{1}=y$, we have

$$
\begin{aligned}
x^{\prime \prime} & =x_{1}+\gamma_{1, \jmath}^{-1} y_{1}^{a_{1}}-h\left(y_{1}\right) \\
y^{\prime \prime} & =y-\gamma_{m, l} x^{b_{m}}+h_{l}(x) \\
& =y_{1}-\gamma_{m, l}\left(x_{1}+\gamma_{1, j}^{-1} y_{1}^{a_{1}}-h\left(y_{1}\right)\right)^{b_{m}}+h_{l}\left(x_{1}+\gamma_{1, \jmath}^{-1} y_{1}^{a_{1}}-h\left(y_{1}\right)\right) \\
& = \begin{cases}y_{1}-\gamma_{m, l} x_{1}^{b} m+h^{\prime}\left(x_{1}, y_{1}\right), \quad m>1 \\
\left(1-\gamma_{1, l} \gamma_{1, j}^{-1}\right) y_{1}-\gamma_{1, l} x_{1}+h^{\prime}\left(x_{1}, y_{1}\right), \quad m=1,\end{cases}
\end{aligned}
$$

where val $h^{\prime}>b_{m}$. In the case of $m=1$ and $a_{1}=1, P_{1}={ }^{t}(1,1)$. By implicit function theorem, there exists an analytic function $H\left(x_{1}\right)$ with val $H>b_{m}$ and a unit $U$ such that

$$
y^{\prime \prime}=y-\gamma_{m, l} x^{b_{m}}+h_{l}(x)=\left\{\begin{array}{l}
U \times\left(y_{1}-\gamma_{m, l} x_{1}^{b} m+H\left(x_{1}\right)\right), \quad m>1 \\
U \times\left(\left(1-\gamma_{1, l} \gamma_{1, \jmath}^{-1}\right) y_{1}-\gamma_{1, l} x_{1}+H\left(x_{1}\right)\right), \quad m=1 .
\end{array}\right.
$$

Thus by the assumption we see that

$$
\begin{aligned}
& \left(y_{1}-\gamma_{m, l} x_{1}^{b} m+H\left(x_{1}\right)\right)^{\nu} m, l \mid f_{1}\left(x_{1}, y_{1}\right), \quad m>1 \\
& \left(\left(1-\gamma_{1, l} \gamma_{1, j}^{-1}\right) y_{1}-\gamma_{1, l} x_{1}+H\left(x_{1}\right)\right)^{\nu_{1}, l} \mid f_{1}\left(x_{1}, y_{1}\right), \quad m=1, P={ }^{t}(1,1) .
\end{aligned}
$$

Thus by the above expression of $f_{1, P_{m}}\left(x_{1}, y_{1}\right)$ and Sublemma (1.9), $y_{1}$ is also good for $f$. Q. E.D.

COROLlary (1.10). Let $f(x, y)$ be a given germ of function. Then there 
exists a change of coordinates $\left(x_{1}, y_{1}\right)$ which is a good coordinate system for $f_{1}\left(x_{1}, y_{1}\right)$.

\section{Non-degenerate plane curves}

Consider as above a complex analytic function $f(x, y)$ defined in a neighborhood of $O$. We define (cf. $[\mathrm{K}],[\mathrm{V}],[\mathrm{O} 1]$ ):

DEFINITION (2.1). Let $\Delta$ be a compact face of the Newton boundary of the function $f$. The function $f$ is non-degenerate on $\Delta$ if the quasi-homogeneous function $f_{\Delta}$ has no critical point in the torus $\left(C^{*}\right)^{2}$. The function $f$ is nondegenerate in the system of coordinates $(x, y)$ if, for any compact face of its Newton boundary, the function $f$ is non-degenerate on this compact face.

Let $\Delta_{1}, \cdots, \Delta_{m}$ be the faces of $\Gamma(f ; x, y)$ and let $P_{1}={ }^{t}\left(a_{1}, b_{1}\right), \cdots, P_{m}=$ ${ }^{t}\left(a_{m}, b_{m}\right)$ be the corresponding weights. Let $P_{\imath}={ }^{t}\left(a_{\imath}, b_{i}\right)$ and

$$
f_{\Delta_{\imath}}(x, y)=f_{P_{i}}(x, y)=c_{\imath} x^{r_{\imath}} y^{s_{i}} \prod_{\jmath=1}^{k_{\imath}}\left(y^{a_{\imath}}-\gamma_{\imath, \jmath} x^{b_{i}}\right)^{\nu_{i, \jmath}}
$$

where $c_{2}, \gamma_{i, 1}, \cdots, \gamma_{\imath, k}$ are non-zero complex numbers and $\nu_{i, 1}, \cdots, \nu_{i, k_{2}}$ (resp. $r_{\imath}$ and $s_{\imath}$ ) are positive (resp. non-negative) integers and $\gamma_{\imath, 1}, \cdots, \gamma_{\imath, k}$ are assumed to be mutually distinct. Then $f$ is non-degenerate on the face $\Delta_{\imath}$ if and only if $\nu_{i, 1}=\cdots=\nu_{i, k_{\imath}}=1$. This can be proved easily using the Euler equality:

$$
d\left(P_{\imath} ; f\right) f_{P_{i}}(x, y)=x \frac{\partial f_{P_{i}}}{\partial x}(x, y)+y \frac{\partial f_{P_{2}}}{\partial y}(x, y)
$$

Now it is convenient to introduce the following notion of quasi non-degeneracy which is motivated by Sublemma (1.9).

DEFINITION (2.2). We say that $f$ is quasi non-degenerate if for any $i, j$ with $\nu_{i, \jmath}>1$, there exists a germ of analytic function $h_{\imath, j}(x, y)$ with $d\left(P_{\imath} ; h_{\imath, \jmath}\right)$ $>a_{i} b_{i}$ such that $\left(y^{a_{\imath}}-\gamma_{\imath, j} x^{b_{i}}+h_{\imath, j}(x, y)\right)^{\nu_{i, j}}$ divides $f(x, y)$.

We will show later that if $f$ is non-degenerate, $f$ is also quasi non-degenerate (see Lemma (2.8) and Lemma (2.9)).

The non-degeneracy of a function depends on the choice of coordinate, but we shall observe in Corollary 3.13 that the quasi non-degeneracy does not depend on the choice of good coordinates. However, for a reduced function germ $f$, the notion of non-degeneracy is also independent of the choice of good coordinates.

In connection with quasi-good coordinates we have the following Lemma:

LEMMA (2.3). Suppose that $f$ is quasi non-degenerate (resp. non-degenerate) with respect to the given coordinates $(x, y)$. Assume that $x$ is a bad coordinate 
for $f$ and assume that

$$
f_{P_{1}}=c_{1} y^{s_{1}}\left(y^{a_{1}}-\gamma_{1,1} x\right)^{\nu_{1,1}} \quad\left(\nu_{1,1}=1 \text { if } f \text { is non-degenerate }\right)
$$

Let $x=x_{1}+\gamma_{1,1}^{-1} y_{1}^{a_{1}}+h\left(y_{1}\right), y=y_{1}$ with val $h>a_{1}$ and assume that $x_{1}$ is a quastgood coordinate system for

$$
f_{1}\left(x_{1}, y_{1}\right):=f\left(x_{1}+\gamma_{1,1}^{-1} y_{1}^{a_{1}}+h\left(y_{1}\right), y_{1}\right)
$$

as guaranteed by Lemma (1.3). Then $f_{1}$ is also quasi non-degenerate (resp. nondegenerate) in the new system of coordinates $\left(x_{1}, y_{1}\right)$.

A similar assertion is true for $y$ coodinate.

Proof. Suppose

$$
f_{P_{1}}=c_{1} y^{s_{1}}\left(y^{a_{1}-\gamma_{1,1}} x\right)^{\nu_{1,1}}
$$

$\left(r_{1}=0\right.$ and $\left.k_{1}=1\right)$ and perform a change of coordinate using Lemma (1.3):

$$
x=x_{1}+\gamma_{1,1}^{-1} y_{1}^{a_{1}}+h\left(y_{1}\right), \quad y=y_{1}, \quad \text { with val } h>a_{1}
$$

so that $x_{1}$ is quasi-good for $f_{1}\left(x_{1}, y_{1}\right):=f\left(x_{1}+\gamma_{1,1}^{-1} y_{1}^{a_{1}}+h\left(y_{1}\right), y_{1}\right)$.

Sublemma (2.3.1). Assume that $\nu_{1,1}=1$. Then $x_{1}$ is quasi-good for $f_{1}\left(x_{1}, y_{1}\right)$ if and only if $x_{1}$ divides $f_{1}\left(x_{1}, y_{1}\right)$.

Proof. Sufficiency is obvious by definition. Therefore we prove that the condition is necessary. Note that the first interior vertex of $\Gamma(f ; x, y)$ is $\left(1, s_{1}\right)$. Thus $\Gamma\left(f_{1} ; x_{1}, y_{1}\right)$ can have at most one face in $\{u \leqq 1\}$. If $x_{1}$ does not divide $f_{1}, \Gamma\left(f_{1} ; x_{1}, y_{1}\right)$ has a unique face $\Delta_{1}^{\prime}$ in $\{u \leqq 1\}$ and we can write $f_{1 \Delta_{1}^{\prime}}\left(x_{1}, y_{1}\right)=c_{1}^{\prime} y_{1}^{s_{1}}\left(y_{1}^{a_{1}^{\prime}}-\gamma^{\prime} x_{1}\right)$ which shows that $x_{1}$ is not quasi-good for $f_{1}$.

Assume that $f$ is non-degenerate with respect to $(x, y)$. Then $\nu_{1,1}=1$. By Assertion (1.5), we have

$$
\Gamma\left(f_{1} ; x_{1}, y_{1}\right) \cap\{u \geqq 1\}=\Gamma(f ; x, y) \cap\{u \geqq 1\}
$$

and $f_{P_{i}}\left(x^{\prime}, y^{\prime}\right)=f_{P_{\imath}}^{\prime}\left(x^{\prime}, y^{\prime}\right)$ for $i \geqq 2$. This observation and Sublemma (2.3.1) imply that

$$
\Gamma\left(f_{1} ; x_{1}, y_{1}\right) \cap\{u \leqq 1\}=\left\{\left(1, s_{1}\right)\right\}
$$

so $f_{1}$ is non-degenerate with respect to $\left(x_{1}, y_{1}\right)$. This proves Lemma (2.3) if $f$ is non-degenerate.

It remains to consider the case $f$ is quasi non-degenerate. We need:

ASSERTION (2.3.2). Assume that $\left(x-\gamma y^{a}+h(x, y)\right)^{\nu}$ divides $f(x, y)$ where $h(x, y)$ is a germ of an analytic function with $d(P ; h)>a$ and $P={ }^{t}(a, 1)$. Then there exists a germ of analytic function $h^{\prime}(y)$ such that val $h^{\prime}>a$ and $\left(x-\gamma y^{a}+\right.$ $\left.h^{\prime}(y)\right)^{\nu}$ divides $f(x, y)$. 
Proof. By the implicit function theorem, we can find an analytic function $h^{\prime}(y)$ so that

$$
x-\gamma y^{a}+h(x, y)=U \times\left(x-\gamma y^{a}+h^{\prime}(y)\right)
$$

where $U$ is a unit with $U(0,0)=1$. As we have

$$
x-\gamma y^{a}=\left(x-\gamma y^{a}+h(x, y)\right)_{P}=U_{P}\left(x-\gamma y^{a}+h^{\prime}(y)\right)_{P}
$$

and $U_{P}=1$, we have

$$
\left(x-\gamma y^{a}+h^{\prime}(y)\right)_{P}=x-\gamma y^{a}
$$

This implies that val $h^{\prime}>a$ and $\left(x-\gamma y^{a}+h^{\prime}(y)\right)^{\nu}$ divides $f$.

As $f(x, y)$ is assumed to be quasi non-degenerate, there exists a germ of an analytic function $h_{1,1}(x, y)$ such that $\left(x-\gamma_{1,1}^{-1} y^{a_{1}}+h_{1,1}(x, y)\right)^{\nu_{1,1}}$ divides $f(x, y)$. Thus by the Assertion (2.3.2) there exists a germ of an analytic function $h_{1,1}^{\prime}(y)$ such that $\left(x-\gamma_{1,1}^{-1} y^{a_{1}}+h_{1,1}^{\prime}(y)\right)^{\nu_{1,1}}$ divides $f(x, y)$. Now consider the following change of coordinate given by:

$$
x^{\prime}=x-\gamma_{1,1}^{-1} y^{a_{1}}+h_{1,1}^{\prime}(y) \text { and } y^{\prime}=y
$$

and denote $f^{\prime}\left(x^{\prime}, y^{\prime}\right):=f\left(x^{\prime}+\gamma_{1,1}^{-1} y^{\prime a_{1}}-h_{1,1}^{\prime}\left(y^{\prime}\right), y^{\prime}\right)$. Obviously $x^{\prime \nu_{1,1}}$ divides $f^{\prime}\left(x^{\prime}, y^{\prime}\right)$. Assume that $\left(x_{1}, y_{1}\right)$ be as above and assume that $x_{1}$ is quasi-good for $f_{1}\left(x_{1}, y_{1}\right)$. By Assertion (1.9.1), we must have $x_{1}=x^{\prime}$ and $y_{1}=y^{\prime}$. It remains to prove that $f_{1}$ is quasi non-degerate with respect to $\left(x_{1}, y_{1}\right)$. As $x_{1}^{\nu_{1}, 1}$ divides $f_{1}\left(x_{1}, y_{1}\right)$, we have

$$
\Gamma\left(f_{1} ; x_{1}, y_{1}\right) \cap\left\{u \geqq \nu_{1,1}\right\}=\Gamma(f ; x, y) \cap\left\{u \geqq \nu_{1,1}\right\}
$$

and $f_{P_{i}}\left(x_{1}, y_{1}\right)=f_{1 P_{i}}\left(x_{1}, y_{1}\right)$ for $i \geqq 2$. Assume that $\nu_{i, j}>1$ for some $i>1$ and $1 \leqq j \leqq k_{\imath}$. Then by the quasi non-degeneracy of $f(x, y)$, there exists a germ of analytic function $h_{\imath, j}(x, y)$ with $d\left(P_{\imath} ; h_{\imath, \jmath}\right)<a_{i} b_{\imath}$ such that $\left(y^{a_{\imath}}-\gamma_{\imath, \jmath} x^{b_{i}}+\right.$ $\left.h_{\imath, j}(x, y)\right)^{\nu_{i, j}}$ divides $f(x, y)$.

As $\left(x_{1}+\gamma_{1,1}^{-1} y_{1}^{a_{1}}-h\left(y_{1}\right)\right)_{P_{2}}=x_{1}$, we have

$$
\begin{aligned}
y^{a_{\imath}}-\gamma_{\imath, \jmath} x^{b_{i}}+h_{\imath, j}(x, y) & =y_{1}^{a_{\imath}}-\gamma_{\imath, j}\left(x_{1}+\gamma_{1,1}^{-1} y_{1}^{a_{1}}-h\left(y_{1}\right)\right)^{b_{i}}+h_{\imath, j}\left(x_{1}+\gamma_{1,1}^{-1} y_{2}^{a_{1}}-h\left(y_{1}\right), y_{1}\right) \\
& =y_{1}^{a_{\imath}}-\gamma_{\imath, \jmath} x_{1}^{b_{i}}+h^{\prime}\left(x_{1}, y_{1}\right)
\end{aligned}
$$

where $d\left(P_{2} ; h^{\prime}\right)>a_{i} b_{i}$. Therefore $\left(y_{1}^{a}{ }^{a}-\gamma_{\imath,}, x_{1}^{b_{i}}+h^{\prime}\left(x_{1}, y_{1}\right)\right)^{\nu_{i, \jmath}}$ divides $f_{1}\left(x_{1}, y_{1}\right)$. Thus $f_{1}$ is quasi non-degenerate with respect to $\left(x_{1}, y_{1}\right)$. A similar proof works for the coordinate $y$. Q.E.D.

The Lemma (2.3) implies:

COROLLARY (2.4). Suppose that $f$ is quasi non-degenerate (resp. non-degenerate) with respect to the given coordinates $(x, y)$. Then there exists a change of 
coordinates $\left(x_{1}, y_{1}\right)$ so that $f$ is quasi non-degenerate (resp. non-degenerate) in $\left(x_{1}, y_{1}\right)$ and $\left(x_{1}, y_{1}\right)$ is a quasi-good system of coordinates of $f$.

Proof. First assume that both coordinates $x$ and $y$ are bad under the condition (2) of (1.1), i.e. we have

$$
f_{P_{1}}(x, y)=c_{1}\left(y-\gamma_{1,1} x\right)^{\nu_{1,1}}\left(y-\gamma_{1,2} x\right)^{\nu_{1}, 2}
$$

where $\nu_{1,1}=\nu_{1,2}=1$ if $f$ is non-degenerate.

Assume first that $f$ is non-degenerate. So we have $\nu_{1,1}=\nu_{1,2}=1$. We first take the coordinate change

$$
x^{\prime}=y-\gamma_{1,1} x, \quad y^{\prime}=y-\gamma_{1,2} x
$$

Then $f^{\prime}\left(x^{\prime}, y^{\prime}\right):=f(x, y)$ is automatically non-degenerate with respect to $\left(x^{\prime}, y^{\prime}\right)$ and we come to the situation as in the condition (1) of (1.1). Applying Lemma (2.3) and Sublemma (2.3.1), we find coordinates $\left(x_{1}, y_{1}\right)$ such that $f_{1}$ is simply the monomial $c x_{1} y_{1}$ and is therefore non-degenerate. (In particular $f$ has a normal crossing singularity at $O$.)

In the case $f$ is quasi non-degenerate, we take the coordinates

$$
x_{1}=y-\gamma_{1,1} x+h_{1,1}(x, y) \text { and } y_{1}=y-\gamma_{1,2} x+h_{1,2}(x, y)
$$

where $h_{1,1}, h_{1,2}$ are chosen so that $\left(y-\gamma_{1,1} x+h_{1,1}(x, y)\right)^{\nu_{1,1}}$ divides $f,\left(y-\gamma_{1,2} x+\right.$ $\left.h_{1,2}(x, y)\right)^{\nu}{ }_{1,2}$ divides $f$ and $d\left(P ; h_{1, \imath}\right)>1$ for $i=1,2$ with $P={ }^{t}(1,1)$. Then $f_{1}$ becomes simply the product $c_{1} x_{1,1}^{\nu_{1}, y_{1,2}^{\nu}}$ and $f_{1}$ has a normal crossing singularity. Now assume that the coordinate $x$ is bad under the condition (1) of (1.1), then we can apply Lemma (2.3) to obtain a quasi-good coordinate $\left(x_{1}, y_{1}\right)$ in which $f_{1}$ is quasi non-degenerate (resp. non-degenerate). Similarly we do the same reasoning for the coordinate $y$. Q.E.D.

LEMMA (2.5). If $(x, y)$ is a quasi-good coordinate system for a function $f(x, y)$ and assume that $f$ is non-degenerate (resp. quasi non-degenerate) in this coordinates. Then the system of coordinates $(x, y)$ is also good for $f$.

Proof. We may assume that $f$ has not a normal crossing singularity at $O$. As the non-degeneracy implies the quasi non-degeneracy by Lemma (2.8) below, we may prove the assertion in the case $f$ is quasi non-degenerate. Let

$$
f_{P_{1}}(x, y)=c_{1} x^{r_{1}} y^{s_{1}} \prod_{\jmath=1}^{k_{1}}\left(y^{a_{1}}-\gamma_{1, \jmath} x^{b_{1}}\right)^{\nu_{1, \jmath}}
$$

If $b_{1}>1$, the assertion follows from the definition. Assume that $b_{1}=1$. As $x$ is quasi-good, either (a) $r_{1}>0$ or (b) $r_{1}=0$ and $k_{1}>1$. In any case, $r_{1}+k_{1} \geqq 2$. By the assumption, $f(x, y)$ is quasi non-degenerate. Using Assertion (2.3.2), we can take a germ of analytic function $h_{1, j}^{\prime}(y)$ such that val $h_{1, j}^{\prime}(y)>a_{1}$ and $\left(x-\gamma_{1, j}^{-1} y^{a_{1}}+h_{1, j}^{\prime}(y)\right)^{\nu_{1, j}}$ divides $f(x, y)$. Let 


$$
X=x-\gamma_{1, j}^{-1} y^{a_{1}}+h_{1, j}^{\prime}(y), \quad Y=y
$$

and let $F(X, Y):=f\left(X+\gamma_{1, j}^{-1} Y^{a_{1}}-h_{1, j}^{\prime}(Y), Y\right)$. Then $X^{\nu_{1, \jmath}}$ divides $F(X, Y)$. Let

$$
x^{\prime}=x-\gamma_{1, j}^{-1} y^{a_{1}}+h(y), \quad y^{\prime}=y
$$

be an arbitrary change of coordinates with val $h>a_{1}$. Let $f^{\prime}\left(x^{\prime}, y^{\prime}\right):=f\left(x^{\prime}+\right.$ $\left.\gamma_{1, j}^{-1} y^{\prime a_{1}}-h\left(y^{\prime}\right), y^{\prime}\right)$. Assume that $x^{\prime}$ is a quasi-good coordinate for $f^{\prime}$. By Assertion (1.9.1), this implies that $x^{\prime}=X$. This implies that $x^{\prime \nu_{1, \jmath}}$ divides $f^{\prime}\left(x^{\prime}, y^{\prime}\right)$. Therefore $x$ is good by Sublemma (1.9). The same argument applies for $y$. Q.E.D.

(2.6). Now we recall the definition of a toroidal modification. We have already introduced the dual Newton diagram $\Gamma^{*}(f ; x, y)$ and we have identified it with a subdivision $R_{-\infty}, P_{1}, \cdots, P_{m}, R_{0}$ of the segment $\left[R_{-\infty}, R_{0}\right]$. For convenience, we denote $P_{0}=R_{-\infty}$ and $P_{m+1}=R_{0}$. Assume that

$$
\operatorname{det}\left(P_{\imath}, P_{\imath+1}\right)=\operatorname{det}\left(\begin{array}{cc}
a_{\imath} & a_{\imath+1} \\
b_{i} & b_{i+1}
\end{array}\right)>1 .
$$

We use Lemma (3.6) of [O3] to obtain a unique regular subdivision $\left[P_{\imath}, P_{\imath, 1}, \cdots\right.$, $\left.P_{\imath, l}, P_{\imath+1}\right]$ so that $\operatorname{det}\left(P_{\imath, \jmath}, P_{\imath, \jmath+1}\right)=1$ for $0 \leqq i \leqq m$ and $0 \leqq j \leqq l_{2}$ (where $P_{\imath, 0}=P_{\imath}$ and $\left.P_{2, l_{i+1}}=P_{2+1}\right)$. Thus we obtain a canonical regular simplicial subdivision $\Sigma^{*}(f ; x, y)$ of $\Gamma^{*}(f ; x, y)$. Let ${ }^{t}\left(a_{\imath, j}, b_{i, j}\right)$ be the unique primitive integral weight vector in the class represented by the point $P_{\imath,}$ (we identify the point $P_{\imath, 3}$ and the unique primitive integral vector contained in it) and let

$$
m_{2, \jmath}=\frac{\left(a_{2, \jmath-1}+a_{2, \jmath+1}\right)}{a_{2, \jmath}}
$$

for any $(i, j)$ such that $0 \leqq i \leqq m, 0 \leqq j \leqq l_{2}$ except $(i, j)=(0,0)$ and with the convention $(i,-1)=\left(i-1, l_{2-1}\right)$. This subdivision has the following properties: the number $m_{\imath,}$, is a positive integer for any $(i, j)$ such that $0 \leqq i \leqq m, 0 \leqq \jmath \leqq l_{\imath}$ and $m_{\imath, \jmath} \geqq 2$ for $0 \leqq i \leqq m, 1 \leqq j \leqq l_{\imath}$. In particular, only $m_{\imath, 0}(i=1, \cdots, m)$ can be possibly 1 . Let

$$
\sigma=\left(\begin{array}{ll}
\alpha & \beta \\
\gamma & \delta
\end{array}\right)
$$

be a unimodular integral $2 \times 2$ matrix. This matrix defines a birational morphism

$$
\pi_{\sigma}:\left(C^{*}\right)^{2} \longrightarrow\left(C^{*}\right)^{2}
$$

by $\pi_{\sigma}(x, y)=\left(x^{\alpha} y^{\beta}, x^{\gamma} y^{\delta}\right)$. If the integers $\alpha$ and $\gamma$ are non-negative, this map can be extended to the axis $\{x=0\}$. Similarly if the integers $\beta$ and $\delta$ are nonnegative, this map can be extended to the axis $\{y=0\}$. Because of the unimodularity of the matrix $\sigma$, the map $\pi_{\sigma}$ is in fact an analytic isomorphism. Note that if $\tau$ is another unimodular $2 \times 2$ matrix

$$
\pi_{\sigma} \circ \pi_{\tau}=\pi_{\sigma \tau} \text { and }\left(\pi_{\sigma}\right)^{-1}=\pi_{\sigma^{-1}}
$$


For each segment $\Sigma_{i, j}=\left[P_{\imath, \jmath}, P_{\imath, \jmath+1}\right]$ and the corresponding unimodular matrix

$$
\sigma_{2,3}:=\left(\begin{array}{ll}
a_{2,3} & a_{2, \jmath+1} \\
b_{i, 3} & b_{i, \jmath+1}
\end{array}\right),
$$

we associate a two dimensional affine space $C_{\sigma_{i}, j}^{2}$ with coordinates $\left(x_{\sigma_{i, j}}, y_{\sigma_{i, j}}\right)$ and the map

$$
\pi_{\sigma_{i, j}}: C_{\sigma_{i, j}}^{2} \longrightarrow C^{2} \text {. }
$$

We use the maps $\pi_{\sigma_{i}}$, to build-up a non-singular algebraic variety $X$ as follows. First we consider the disjoint union $\cup_{\imath,}, C_{\sigma_{i},}^{2}$ and the variety $X$ is the quotient of this union obtained by identifying points $\left(x_{\sigma_{i, j}}, y_{\sigma_{i, j}}\right) \in C_{\sigma_{i, j}}^{2}$ and $\left(x_{\sigma_{k}, l}, y_{\sigma_{k}, l}\right)$ $\in C_{\sigma_{k}, l}^{1}$ if and only if the birational map $\pi_{\sigma_{k, l}^{-1} \sigma_{i, j}}$ is defined at the point $\left(x_{\sigma_{i, j},}, y_{\sigma_{i, j}}\right)$ and

$$
\pi_{\sigma_{k, l}^{-1} \sigma_{i, j}}\left(x_{\sigma_{i, j}}, y_{\sigma_{i, j}}\right)=\left(x_{\sigma_{k}, l}, y_{\sigma_{k, l}}\right) .
$$

Again by the unimodularity of the above matrices, $X$ is non-singular. The maps $\left\{\pi_{\sigma_{i}, j}\right\}$ glue into a proper birational algebraic map $\pi: X \rightarrow C^{2}$.

DEFINITION (2.7). The map $\pi$ is called the toroidal modification associated to (the canonical regular simplicial subdivision $\Sigma^{*}(f ; x, y)$ of) the dual Newton diagram $\Gamma^{*}(f ; x, y)([\mathrm{V}],[\mathrm{O} 3,4])$.

Note that we only consider regular toroidal modification. In the sequence when we speak about the toroidal modification associated to $(x, y)$, we implicitly deal with an analytic function $f$ and we mean the toroidal modification associated to the canonical regular simplicial subdivision $\Sigma^{*}(f ; x, y)$ of the dual Newton diagram $\Gamma^{*}(f ; x, y)$.

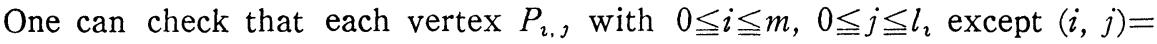
$(0,0)$ defines a component of the exceptional divisor of $\pi$ which we denote by $\hat{E}\left(P_{\imath, \jmath}\right)$ and which is a non-singular rational curve. In the coordinate chart $C_{\sigma_{i}, j}^{2}, \hat{E}\left(P_{\imath, \jmath}\right)$ is defined by $x_{\sigma_{i, j}}=0$. Actually we have

$$
\hat{E}\left(P_{\imath, \jmath}\right)=\left(\boldsymbol{C}_{\sigma_{i, j}}^{2} \cap\left\{x_{\sigma_{i}, j}=0\right\}\right) \cup\left(\boldsymbol{C}_{\sigma_{i, j-1}}^{2} \cap\left\{y_{\sigma_{i, j-1}}=0\right\}\right) .
$$

By analogy we denote by $\hat{E}\left(P_{0}\right)$ and $\hat{E}\left(P_{m+1}\right)$ the non-compact components

$$
\hat{E}\left(P_{0}\right)=C_{\sigma_{0,0}}^{2} \cap\left\{x_{\sigma_{0,0}}=0\right\}, \quad \hat{E}\left(P_{m+1}\right)=C_{\sigma_{m}, l_{m}}^{2} \cap\left\{y_{\sigma_{m, l}}=0\right\}
$$

which are isomorphically mapped on the axis $\{x=0\}$ and $\{y=0\}$. The selfintersection numbers $\hat{E}\left(P_{\imath, j}\right)^{2}$ can be easily computed by the well-known property (see for example [La], Theorem (2.6)):

$$
(\pi *(x)) \cdot \hat{E}\left(P_{\imath, j}\right)=0
$$

where $\left(\pi^{*}(x)\right)$ is the divisor associated to the function $\pi *(x)=x \circ \pi$. Note that 


$$
\left(\pi^{*}(x)\right)=\sum_{\imath, j} a_{\imath, j} \hat{E}\left(P_{\imath, j}\right)+\hat{E}\left(P_{0}\right)
$$

Therefore we can see that $\hat{E}\left(P_{\imath, j}\right)^{2}=-m_{\imath, \jmath}$ (see $[\mathrm{O} 2,3]$ ).

On the other hand, in the chart $C_{\sigma_{i},}^{2}$, the restriction of the function $\pi^{*} f$ is given by:

$$
\begin{aligned}
& \pi^{*} f\left(x_{\sigma_{i, 0},}, y_{\sigma_{\imath, 0}}\right)=c_{\imath} x_{\sigma_{i, 0}}^{d\left(P_{i, f}\right)} y_{\sigma_{\imath, 0}}^{d\left(P_{i, 1}, f\right)}\left(\prod_{\jmath=1}^{k_{2}}\left(y_{\sigma_{i, 0}}-\gamma_{\imath, j}\right)^{\nu_{i, \jmath}}+x_{\sigma_{i, 0}} y_{\sigma_{i, 0}} \Psi\right) \\
& \pi * f\left(x_{\sigma_{i, l},}, y_{\sigma_{i, l}}\right)=c_{\imath} x_{\sigma_{i, l}}^{d\left(P_{i, l} ; f\right)} y_{\sigma_{i, l}}^{d\left(P_{i, l+1}, f\right)}\left(\prod_{\jmath=1}^{k_{2}}\left(-\gamma_{\imath, j}\right)^{\nu_{i, \jmath}}+x_{\sigma_{i, l}} y_{\sigma_{i, l}} \Psi\right)
\end{aligned}
$$

where $\Psi$ is a germ of analytic function. Let

$$
f_{\sigma_{i, l}}\left(x_{\sigma_{i, l},}, y_{\sigma_{i, l}}\right)= \begin{cases}\pi * f\left(x_{\sigma_{i, l}}, y_{\sigma_{i, l}}\right) / x_{\sigma_{i} l}^{d\left(P_{i, l} ; f\right)} y_{\sigma_{i, l}}^{d\left(P_{i, l+1}, f\right)} & (i, l) \neq(0,0),\left(m, l_{m}\right) \\ \pi^{*} f\left(x_{\sigma_{0,0},}, y_{\sigma_{0,0}}\right) / y_{\sigma_{0}, 0}^{d\left(P_{i, 1}, f\right)} & (i, l)=(0,0) \\ \pi^{*} f\left(x_{\sigma_{m}, l_{m}}, y_{\sigma_{m, l}}\right) / x_{\boldsymbol{\sigma}_{m}, l m}^{d\left(\boldsymbol{P}_{m, l m}, f\right)} & (i, l)=\left(m, l_{m}\right) .\end{cases}
$$

Obviously $f_{\sigma_{i, l}}=0$ is the defining equation of the strict transform of $\{f=0\} \cap C_{\sigma_{i, l}}^{2}$. So the strict transform of $\{f=0\}$ only intersects with $\hat{E}\left(P_{1}\right), \cdots, \hat{E}\left(P_{m}\right) . \hat{E}\left(P_{0}\right)$ (resp. $\hat{E}\left(P_{m+1}\right)$ ) is a component of this strict transform if $x$ (resp. $y$ ) divides $f$.

Let $C_{2,}$ be the germ of the curve at the origin which is the image of the germ of the curve $\tilde{C}_{\imath, j}:=\left\{f_{\sigma_{i, 0}}\right\}=0$ at $\left(0, \gamma_{\imath, j}\right) \in \boldsymbol{C}_{\sigma_{i, 0}}$. For simplicity, we denote the point represented by $\left(0, \gamma_{\imath, \jmath}\right) \in C_{\sigma_{i, 0}}$ by $\xi_{\imath, \jmath}$. In general, $C_{\imath, \jmath}$ is not reduced and a union of same irreducible components of $C=\{f=0\}$ with positive integers as coefficients. The following lemma gives the form of the equations of $C_{\imath, 3}$ and the irreducible components $C_{\imath, \jmath, k}$ of $C_{\imath, \jmath}$ :

LEMMA (2.8). (1) Let $r$ be the number of irreducible camponents of $C=$ $\{f=0\}$. Then $r \geqq \sum_{\imath=1}^{m} k_{\imath} \geqq m$. In partıcular, if $C$ is irreducible, we must have $m=0$ or $m=1$ and the Newton boundary touches to the both axis.

(2) Let $C_{\imath, \jmath}=n_{\imath, \jmath, 1} C_{\imath, \jmath, 1}+\cdots+n_{\imath, \jmath, t_{2, j}} C_{\imath, \jmath, t_{\imath, \jmath}}$ where $C_{\imath, \jmath, 1}, \cdots, C_{\imath, \jmath, t_{\imath, \jmath} \text { are }}$ distinct irreducible components. Let $f_{\imath, j}(x, y)$ and $f_{\imath, \jmath, k} k=1, \cdots, t_{\imath, j}$ be the defining functions of $C_{\imath, \jmath}$ and $C_{\imath, 3, k}$ respectively. Then multiplying by a unit if necessary, we can write as

$$
\begin{cases}f_{\imath, j}(x, y)=\left(y^{a_{\imath}}-\gamma_{\imath, \jmath} x^{b_{i}}\right)^{\nu_{i, \jmath}}+h_{\imath, j}(x, y), & d\left(P_{\imath} ; h_{\imath, j}\right)>a_{i} b_{i} \nu_{i, \jmath} \\ f_{\imath, \jmath, k}(x, y)=\left(y^{a_{\imath}}-\gamma_{\imath, \jmath} x^{b_{\imath}}\right)^{\nu_{i, \jmath, k}}+h_{\imath, \jmath, k}(x, y), & d\left(P_{\imath} ; h_{\imath, \jmath, k}\right)>a_{i} b_{i} \nu_{i, \jmath, k}\end{cases}
$$

and $\sum_{k=1}^{t_{\imath, \jmath}} n_{\imath, \jmath, k} \nu_{i, \jmath, k}=\nu_{i, \jmath}$.

Proof. The assertion (1) is obvious. We consider the equality

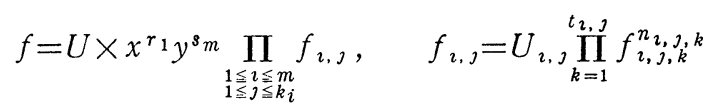




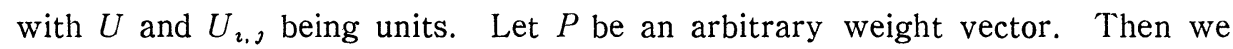
have

$$
f_{P}=U_{P} \prod_{\imath, \jmath}\left(f_{\imath, j}\right)_{P}, \quad\left(f_{\imath, j}\right)_{P}=\left(U_{\imath, \jmath}\right)_{P} \prod_{k=1}^{t_{\imath, j}}\left(f_{\imath, \jmath, k}\right)_{P}^{n_{2, \jmath, k}}
$$

Thus using the assertion (1), $f_{\imath, \jmath, k}(x, y)$ is either $x$ or $y$ or $\left(y^{a} h-\gamma_{h, l} x^{b_{h}}\right)^{\nu} h, l, k$ + (higher terms). However the proper transform of the curve $\left\{\left(y^{a} h-\gamma_{h, l} x^{b_{h}}\right)^{\nu} i, \jmath, k\right.$ $+($ higher terms $)=0\}$ passes through $\xi_{2, j}$ if and only if $(h, l)=(i, j)$. This proves the assertion (2). Q.E.D.

Lemma (2.9). The function $\pi * f$ has a normal crossing singularity at $\xi_{2, j} \in$ $C_{\sigma_{i, 0}}^{2}$ if and only if there exists a germ of a function $h_{\imath, j}(x, y)$ with $d\left(P_{\imath} ; h_{\imath, j}\right)$ $>a_{i} b_{i}$ so that $\left(y^{a_{\imath}}-\gamma_{\imath,}, x^{b_{i}}+h_{\imath, \jmath}(x, y)\right)^{\nu_{i, j}}$ divide $f(x, y)$. (In the notations of Lemma (2.8), this implies $t_{\imath, \jmath}=1, n_{\imath, \jmath, 1}=\nu_{i, j}$.) In particular if $\nu_{i, \jmath}=1, C_{\imath, \jmath}$ is irreducible and $\pi^{*} f$ has a (reduced) normal crossing singularity at $\xi_{2, j}$.

Proof. Let

$$
\sigma_{\imath, 0}:=\left(\begin{array}{ll}
a_{\imath} & a_{\imath i}^{i} \\
b_{i} & b_{i, 1}
\end{array}\right), \quad a_{i} b_{i, 1}-a_{\imath, 1} b_{i}=1
$$

Then by the definition of the mapping $\pi_{\sigma_{i, 0}}: C_{\sigma_{i, 0}}^{2} \rightarrow C^{2}$ and (2.7.1), we have

$$
\begin{gathered}
y^{a_{\imath}}-\gamma_{\imath, j} x^{b_{i}}=x_{\sigma_{i}, 0}^{a_{i} b_{i}} y_{\sigma_{i}, 0}^{a_{2,0} b_{i}}\left(y_{\sigma_{i, 0}}-\gamma_{\imath, j}\right) \quad \text { therefore } \\
\pi * f\left(x_{\sigma_{i, 0},} y_{\sigma_{i, 0}}\right)=c_{\imath} x_{\sigma_{i},}^{d\left(P_{i} ; f\right)} y_{\sigma_{i, 0}}^{d\left(P_{i, 1}, f\right)}\left(\prod_{\jmath=1}^{k_{2}}\left(y_{\sigma_{i, 0}}-\gamma_{\imath, j}\right)^{\nu_{i, j}}+x_{\sigma_{i, 0}} y_{\sigma_{i, 0}} \psi\left(x_{\sigma_{i, 0}}, y_{\sigma_{i, 0}}\right)\right)
\end{gathered}
$$

Take

$$
x^{\prime}=x_{\sigma_{i, 0}}, \quad y^{\prime}=y_{\sigma_{i, 0}}-\gamma_{i, j}
$$

as a system of coordinates centered at $\xi_{\imath, j} \in C_{\sigma_{i, 0}}^{2}$. Note that

$$
\left.\pi * f\right|_{\sigma_{i, 0}^{2}} ^{2}=c_{\imath} x^{\prime d\left(P_{i} ; f\right)} U_{1} \times\left(y^{\prime \nu_{i, j}} U_{2}+x^{\prime} \phi\right)
$$

where $U_{1}, U_{2}$ are units and $\phi$ is a germ of analytic function at $\xi_{2, \jmath}$. We need.

ASSERTION (2.10). The function $\pi^{*} f$ has a normal crossing singularity at $\xi_{2,}$, if and only if there exists an analytic function $h\left(x^{\prime}\right)$ vanishing at $x^{\prime}=0$ so that

$$
y^{\prime \nu_{i, j}} U_{2}+x^{\prime} \psi=\left(y^{\prime}+x^{\prime} h\left(x^{\prime}\right)\right)^{\nu_{i, j}} U_{3}
$$

where $U_{3}$ is a unit and $h\left(x^{\prime}\right)$ is a germ of an analytic function at $\xi_{\imath, \jmath}$.

We assume Assertion (2.10) for a while. Assume first that there exists a germ of a function $h(x, y)$ with $d\left(P_{i} ; h\right)>a_{i} b_{\text {, so that }}\left(y^{a_{\imath}}-\gamma_{\imath,} x^{b_{i}}+h(x, y)\right)^{\nu_{i}, \text { s }}$ divide $f(x, y)$. Then under the same notation as in Lemma (2.8), 
and we can write

$$
f_{\imath, j}(x, y)=\left(y^{a_{\imath}}-\gamma_{\imath, j} x^{b_{i}}+h(x, y)\right)^{\nu_{i, j}}
$$

$$
\pi^{*} f_{\imath, j}\left(x^{\prime}, y^{\prime}\right)=x^{\prime a_{i} b^{\nu^{\nu}} i, \nu}\left(y^{\prime}+x^{\prime} h^{\prime}\left(x^{\prime}, y^{\prime}\right)\right)^{\nu i, j} U
$$

as a function germ at $\xi_{2,3}$ where $h^{\prime}\left(x^{\prime}, y^{\prime}\right)$ is a suitable germ of an analytic function and $U$ is a unit. This implies that

$$
\pi^{*} f\left(x^{\prime}, y^{\prime}\right)=x^{d\left(P_{i} ; f\right)}\left(y^{\prime}+x^{\prime} h^{\prime}\left(x^{\prime}, y^{\prime}\right)\right)^{\nu_{i, j} U^{\prime}}
$$

where $U^{\prime}$ is a unit. Thus $\pi * f$ has a normal crossing singularity at $\xi_{2, \jmath}$.

Conversely assume that $\pi * f$ has a normal crossing singularity at $\xi_{\imath, \jmath}$. By Assertion (2.10), there exists a germ of function $h\left(x^{\prime}\right)$ so that

$$
\pi^{*} f=U x^{\prime d\left(P_{i} ; f\right)}\left(y^{\prime}+x^{\prime} h\left(x^{\prime}\right)\right)^{\nu_{i, j}}
$$

This implies that $\tilde{C}_{\imath, \jmath}=\nu_{i, j} L$ where $L:=\left\{y^{\prime}+x^{\prime} h\left(x^{\prime}\right)=0\right\}$ and $t_{\imath, \jmath}=1$. Thus $C_{\imath, \jmath}=\nu_{i, j} C_{\imath, \jmath, 1}$ in the notation of Lemma (2.8). This also implies that $f_{\imath, \jmath, 1}(x, y)$ $=y^{a_{\imath}}-\gamma_{\imath, j} x^{b_{i}}+h_{\imath, \jmath, 1}(x, y)$ with $d\left(P_{\imath} ; h_{\imath, \jmath, 1}\right)>a_{\imath} b_{i}$ and $f_{\imath, \jmath}=f_{\imath, j, 1}^{\nu}$. As $f_{\imath, j}(x, y)$ divides $f(x, y)$, this proves the assertion. Q.E.D.

Proof of Assertion (2.10). Let $g\left(x^{\prime}, y^{\prime}\right)=c_{2} x^{\prime d\left(P_{i} ; f\right)} U_{1} \times\left(y^{\prime \nu_{\imath}, J} U_{2}+x^{\prime} \phi\right)$. The sufficiency is clear. Assume that the function $g$ has a normal crossing singularity at the origin. Obviously $x^{\prime}=0$ is a component. Thus we can write $g\left(x^{\prime}, y^{\prime}\right)$ $=x^{\prime a} l\left(x^{\prime} y^{\prime}\right)^{b}$ where $l\left(x^{\prime}, y^{\prime}\right)=0$ is the other smooth components. Using the implicit function theorem, we can write $l\left(x^{\prime}, y^{\prime}\right)=U_{4} \cdot\left(y^{\prime}+h\left(x^{\prime}\right)\right)$ with $h(0)=0$. Now considering the equality

$$
c_{2} x^{\prime d\left(P_{i} ; f\right)} U_{1} \times\left(y^{\prime \nu_{i}, J} U_{2}+x^{\prime} \psi\right)=x^{\prime a}\left(U_{4} \cdot\left(y^{\prime}+h\left(x^{\prime}\right)\right)\right)^{b}
$$

we can easily see that $a=d\left(P_{\imath} ; f\right), b=\nu_{i,}$ and

$$
y^{\prime \nu i, j} U_{2}+x^{\prime} \psi=\left(y^{\prime}+h\left(x^{\prime}\right)\right)^{\nu i, j} U_{3}
$$

for some unit $U_{3}$. In particular if $\nu_{\imath, \jmath}=1, \pi * f$ has a normal crossing singularity at $\xi_{\imath, j}$. This proves the assertion.

Example (2.10.1). Let us consider the examples considered in Example (1.2.1). (A) Let

$$
f(x, y)=x\left(x+y^{2}\right)^{2}+x^{4} y
$$

Then $\Gamma^{*}(f ; x, y)$ has only one vertex $P_{1}={ }^{t}(2.1)$. 


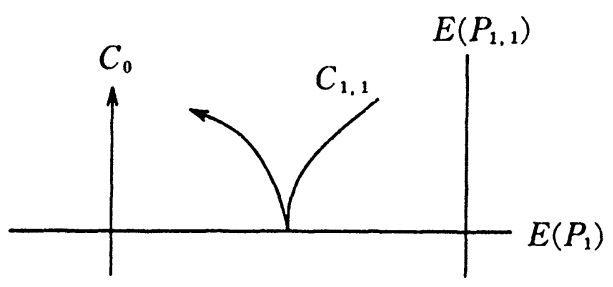

Figure (2.10.1.A)

For the canonical regular subdivision, we need one more vertex $P_{1,1}={ }^{t}(1,1)$. In the toric coordinate chart

$$
\sigma:=\left(P_{1}, P_{1,1}\right)=\left(\begin{array}{ll}
2 & 1 \\
1 & 1
\end{array}\right)
$$

we can write

$$
\pi * f\left(x_{\sigma}, y_{\sigma}\right)=x_{\sigma}^{6} y_{\sigma}^{3}\left(\left(y_{\sigma}+1\right)^{2}+x_{\sigma}^{3} y_{\sigma}^{2}\right)
$$

Thus the strict transform is a cusp at $(0,-1)$.

Now we consider the case (B) in Example (1.2.1):

$$
g(x, y)=\left(x+y^{2}\right)\left(x-y^{2}\right)\left(x-y^{2}+y^{2+m}\right)\left(y+x^{2}\right)
$$

Let $C_{1,1}=\left\{x+y^{2}=0\right\}, C_{1,2}=\left\{x-y^{2}=0\right\}, C_{1,3}=\left\{x-y^{2}+y^{2+m}=0\right\}$ and $C_{2,1}=$ $\left\{y+x^{2}=0\right\}$. Then the strict transforms $\tilde{C}_{1, \imath}$ of $C_{1, \imath}, \imath=1,2,3$ are smooth and intersect transversely with $\hat{E}\left(P_{1}\right)$ and $\widetilde{C}_{1,2}$ and $\tilde{C}_{1,3}$ are tangent with the intersection multiplicity $m$. The Figure (2.10.1.B) shows the situation for $m=2$.

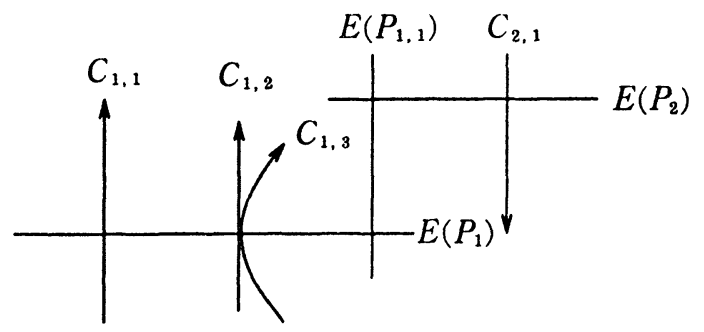

Figure (2.10.1.B)

Recall that a map $p: Y \rightarrow V$ is a resolution of the function $f$ on a neighborhood $V$ of $O$ in $C^{2}$ if :

i) $Y$ is non-singular;

ii) $p$ is a proper analytic mapping and the restriction $p: Y-p^{-1}(O) \rightarrow V-\{0\}$ is biholomorphic ; 
iii) the divisor $\left(p^{*} f\right)$ defined by the pull-back $p^{*} f:=f \circ p$ has only normal crossing singularities and its irreducible components are non-singular.

It is known that each component of the divisor $p^{-1}(O)$ is isomorphic to $\boldsymbol{P}^{1}$. We remark here that the function $f$ need not reduced. To such a resolution of $f$, we associate the dual resolution graph $\mathcal{G}(p)$ of $p$ in the following way: let $E_{2}(1 \leqq i \leqq s)$ be the distinct compact components of the divisor $\left(p^{*} f\right)$ i.e., $p^{-1}(O)=\bigcup_{i=1}^{s} E_{\imath}$ and let $\tilde{C},(1 \leqq j \leqq t)$ be the distinct non-compact components of $\left(p^{*} f\right)$. Namely we have $\left(p^{*} f\right)=\sum_{i=1}^{s} m_{i} E_{i}+\sum_{j=1}^{t} n_{j} \tilde{C}_{\jmath}$. Note that the union of $\tilde{C}$, are the proper transform of $C=\{f=0\}$. To each $E_{\imath}$ we associate a vertex $v_{i}$, an edge joining $v_{\imath}$ and $v_{l}$ if $E_{i} \cap E_{l} \neq \emptyset$. The total dual resolution graph $\mathcal{G}^{\prime}(p ; f)$ of $f$ with respect to $p$ is obtained by adding a vertex $w$, for each $C$, and an edge between $w_{j}$ and $v_{i}$ when $C_{j} \cap E_{i} \neq \emptyset$. The dual resolution graph and the total dual resolution graph are in fact trees and in general they are not bamboos.

Recall that the resolution of the function $f$ in the neighborhood of the origin is minimal if and only if there is no component of the exceptional divisor $p^{-1}(O)$ with self-intersection number -1 which intersects at most two components of the divisor $\left(p^{*} f\right)$. In the case of quasi non-degenerate function, we have :

Proposition (2.11). Assume that the function $f$ is quasi non-degenerate with respect to the coordinates $x, y$ and let $\pi: X-C^{2}$ be the associated toroidal modification. Then $\pi: X \rightarrow C^{2}$ is a resolution of $f$ near the origin. If $(x, y)$ is a quasi-good coordinate system, $\pi: X \rightarrow C^{2}$ is a minimal resolution.

Proof. The first assertion results from Lemma (2.9). We assume that $(x, y)$ is a quasi-good coordinate system and we will show $\pi: X \rightarrow C^{2}$ is minimal. We saw above that the self-intersection numbers of the divisors $\hat{E}\left(P_{\imath, j}\right)$ is equal to $-m_{\imath, \jmath}$. Furthermore for $0 \leqq i \leqq m$ and $1 \leqq \jmath \leqq l_{\imath}$ the integer $-m_{\imath, \jmath} \leqq-2$ (see [O2], Lemma (3.6) of [O3]). Therefore the only components to be checked are $\hat{E}\left(P_{\imath, 0}\right)=\hat{E}\left(P_{\imath}\right)$ for $1 \leqq \imath \leqq m$. If $2 \leqq i \leqq m-1, \hat{E}\left(P_{\imath}\right)$ intersects with $\hat{E}\left(P_{\imath-1, l_{\imath-1}}\right)$, $\hat{E}\left(P_{\imath, 1}\right)$ and at least a non-compact component of the divisor $(\pi * f)$. Therefore this component $\hat{E}\left(P_{2}\right)$ intersects at least three components of the divisor $(\pi * f)$. It remains to consider $\hat{E}\left(P_{1}\right)$ and $\hat{E}\left(P_{m}\right)$. Now we shall see that $x$ and $y$ being quasi-good coordinates, each of $\hat{E}\left(P_{1}\right)$ and $\hat{E}\left(P_{m}\right)$ intersects at least three components of the divisor $\left(\pi^{*} f\right)$. We will see this assertion for $\hat{E}\left(P_{1}\right)$. Let

Recall that

$$
f_{P_{1}}(x, y)=c_{1} x^{r_{1}} y^{s_{1}} \prod_{j=1}^{k 1}\left(y^{a_{1}}-\gamma_{2, j} x^{b_{1}}\right)^{\nu_{1, j}}
$$

$$
p^{*} f \mid \boldsymbol{C}_{\sigma_{1,0}}^{2} \equiv c_{1} x_{\sigma_{1,0}}^{d\left(P_{1}, f\right)} y_{\sigma_{1,0}}^{d\left(P_{1,1}, f\right)} \prod_{j=1}^{k 1}\left(y_{\sigma_{1,0}}-\gamma_{1, j}\right)^{\nu_{i, j}} \quad \bmod \left(x_{\sigma_{1,0}}^{d\left(P_{1}, f\right)+1} y_{\sigma_{1,0}}^{d\left(P_{1,1}, f\right)+1}\right)
$$

Assume first that $m \geqq 2$. Then $\hat{E}\left(P_{1}\right)$ intersects with $\hat{E}\left(P_{1,1}\right)$ and $k_{1}(\geqq 1)$ noncompact components $\widetilde{C}_{1,1}, \cdots, \tilde{C}_{1, k_{1}}$ of $\left(\pi^{*} f\right)$. Either $l_{0}>0$ (this means $\operatorname{det}\left(P_{0}, P_{1}\right)$ 
$>1)$ or $l_{0}=0$. In the first case, $\hat{E}\left(P_{1}\right)$ intersects with $\hat{E}\left(P_{0, l_{0}}\right)$. In the second case, we have $P_{1}={ }^{t}\left(a_{1}, 1\right)$. $x$ being quasi-good, either $k_{1} \geqq 2$ or $k_{1}=1$ and $x$ divides $f$ i.e., $r_{1}>0$. If $x$ divides $f, \hat{E}\left(P_{0}\right)$ is a non-compact component of the divisor $(\pi * f)$ which intersects $\hat{E}\left(P_{1}\right)$. So in any case $\hat{E}\left(P_{1}\right)$ intersects at least three components of the divisor $\left(\pi^{*} f\right)$ and for $\hat{E}\left(P_{m}\right)$ we have similar arguments. Assume now that $m=1$. If $l_{1} \geqq 1$, the argument above applies. If $l_{1}=0$, either $l_{0} \geqq 1$ or $l_{0}=0$. If $l_{0} \geqq 1, \hat{E}\left(P_{1}\right)$ intersects $\hat{E}\left(P_{0, l_{0}}\right)$ and $k_{1}$ non-compact components $\tilde{C}_{1,1}, \cdots, \widetilde{C}_{1, k_{1}}$. $y$ being quasi-good, either $k_{1} \geqq 2$, or $y$ divides $f$ and $\hat{E}\left(P_{m+1}\right)$ is a non-compact component of the divisor $\left(\pi^{*} f\right)$ which also intersects $\hat{E}\left(P_{1}\right)$. Thus in any case $\hat{E}\left(P_{1}\right)$ intersects at least three divisors. If $l_{1}=l_{0}=0$, the map $\pi$ is the blowing-up of the point $O$. This is the case if and only if $m=1$ and $P_{1}=$ ${ }^{t}(1,1)$. By the assumption that $x$ and $y$ are quasi-good coordinates, there are three possible cases: (1) $k_{1} \geqq 3$ or (2) $k_{1}=2$ and $x \mid f$ or $y \mid f$ or (3) $k_{1}=1$ and $x \mid f$ and $y \mid f$. In any cases $\hat{E}\left(P_{1}\right)$ intersects at least three non-compact components of the divisor $(\pi * f)$. Q. E. D.

By a similar argument, we obtain

LEMMA (2.12). Let $f$ be a complex analytic function defined in a neighborhood of the origin in $\boldsymbol{C}^{2}$. Assume that $x$ (resp. $y$ ) be quast-good coordinate for $f$ at the origin and $\pi: X \rightarrow C^{2}$ be the associated toroidal blowing-up. Then compact divisors $\hat{E}\left(P_{\imath}\right)(i=1, \cdots, m-1)$ (resp. $\imath=2, \cdots, m$ ) intersect at least three components of the divisor $(\pi * f)$.

Remark (2.13). Another way to state this Lemma and Proposition (2.11) is to say that, when $f$ is quasi non-degenerate with respect to $(x, y)$ and $(x, y)$ are quasi-good for $f$, the compact divisors $\hat{E}\left(P_{\imath}\right)(i=1, \cdots, m)$ are the rupture components of the minimal resolution of $f$ in the sense of (1.3.10) in [L-M-W], because these divisors intersect at least three components of the divisor $\left(\pi^{*} f\right)$ at normal crossing singularities.

More generally, when $(x, y)$ are quasi-good for an analytic function $f$, the toroidal modification associated to $(x, y)$ will be of a particular interest. Therefore it is reasonable to introduce the following definition:

Definition (2.14). A quasi-good (resp. good) toroidal modification $\pi: X \rightarrow C^{2}$ of $f$ is the toroidal modification associated to the canonical regular subdivision of the dual Newton diagram $\Gamma^{*}(f ; x, y)$ of $f$ with respect to a system of coordinates $(x, y)$ which is quasi-good (resp. good) for $f$. Its restriction over a neighborhood $V$ of the origin is also called a quasi-good (resp. good) toroidal modification.

\section{Resolution Complexity}

Let $\mathscr{F}$ be a graph which is a tree. Let $V(\mathscr{F})$ be the set of vertices of $\mathscr{F}$. For any vertex $v \in V(\mathscr{F})$, let $\delta(v)$ be the number of edges meeting at $v$. 
DEFINITION (3.1). The complexity of the graph $\rho(\mathscr{I})$ is defined by

$$
\rho(\mathscr{I}):=1+\sum_{v \in V(\mathscr{T})} \max (\delta(v)-2,0) .
$$

The graph $\mathscr{F}$ is called a bamboo if $\rho(\mathscr{F})=1$. The resolution complexity $\rho(p ; f)$ of a minimal resolution $p: Y \rightarrow V$ of $f$ at the origin of $f$ is defined by the complexity of the resolution graph $\mathcal{G}(p)$. If $f$ has a normal crossing singularity at $O, \rho(f)=0$ by definition.

Remark (3.2). If $\mathscr{I}$ has at least two vertices, it is easy to see that $\rho(\mathscr{I})$ +1 is equal to the number of vertices $v$ with $\delta(v)=1$. We call such vertices the ends of the graph. The definition of the resolution complexity does not depend on the minimal resolution $p: Y \rightarrow V$ because any two minimal resolutions are isomorphic above a neighborhood of the origin. Thus we denote this number by $\rho(f)$ hereafter.

Let $p: Y \rightarrow V$ be a modification at the origin. Namely $Y$ is smooth, $V$ is a neighborhood of the origin, $p$ is proper and the restriction $p: Y-p^{-1}(O) \rightarrow$ $V-\{O\}$ is biholomorphic. Furthermore assume that the components $E_{1}, \cdots, E_{s}$ of $p^{-1}(O)$ are non-singular curves.

DEFinition (3.3). Let $\xi \in\left(E_{i}-\cup_{j \neq \imath} E_{j}\right)$ and assume that $p^{*} f$ has a singularity at $\xi$ which is not a normal crossing singularity. A system of coordinates $(u, v)$ centered at $\xi$ is called admissible with $p: Y \rightarrow V$ if $u=0$ (or $v=0$ ) is the defining equation of $E_{\imath}$. We say that $(u, v)$ is an admissible quasi-good (resp. admissible good) system of coordinates if it is admissible and $v$ is quasi-good (resp. good), assuming that $u=0$ is the defining equation of $E_{\imath}$.

Note that $u$ is necessarily quasi-good as $u$ divides $p^{*} f$.

DeFinition (3.4). A map $q: Z \rightarrow V$ is a quasi-good (resp. good) toroidal resolution of the function $f(x, y)$ if

(i) the map $q$ is the composition

$$
\pi_{1} \circ \cdots \circ \pi_{k}: X_{k} \rightarrow X_{k-1} \rightarrow \cdots \rightarrow V
$$

of a quasi-good (resp. good) toroidal modification $\pi_{1}$ and non-trivial quasi-good toroidal modifications $\pi_{\jmath}: X_{\jmath} \rightarrow X_{\jmath-1}$ for $j, k \geqq j \geqq 2$ where $X_{k}=Z$ and $\pi_{\jmath}$ is the toroidal modification associated to the function $f \circ \pi_{1} \circ \cdots \circ \pi_{j-1}$ and admissible quasi-good (resp. admissible good) coordinates with the modification $\pi_{1} \circ \cdots \circ \pi_{\jmath-1}$ (in short we shall call these modifications admissible quasi-good (resp. admissible good) toroidal modifications).

(ii) the map $q: Z \rightarrow V$ is a resolution of $f$ in a neighborhood of $O$.

We first prove the existence of a quasi-good toroidal resolution.

Let $p: Y \rightarrow V$ be a modification at the origin. Hereafter $V$ is assumed to be a sufficiently small neighborhood of the origin. 
Denote $E_{1}, \cdots, E_{s}$ the irreducible components of $p^{-1}(O)$. We use the following well-known properties (see [La] Theorem (5.9))

FACT (3.5). The modification $p$ is a finite composition of ordinary blowingups. In particular, the curves $E_{\imath}$ are non-singular and isomorphic to the Riemann sphere $\boldsymbol{P}^{1}$ and there exists an exceptional divisor $E_{\imath}$ with $E_{\imath}^{2}=-1$.

Let $f$ be a complex analytic function defined on a neighborhood of the origin $O$. Let $\widetilde{C}$ be the proper transform of $C=\{f=0\}$ by the modification $p: Y \rightarrow V$ and let $p^{-1}(O) \cap \widetilde{C}=\left\{\xi_{1}, \cdots, \xi_{t}\right\}$. Let $\widetilde{C}$, be the germ of a curve at $\xi$, which is defined by $\widetilde{C}$. The germ $\tilde{C}$, is not necessarily either irreducible or reduced.

Now we assume that $p^{*} f$ has a normal crossing singularity at any nonempty intersections $E_{i} \cap E$, with $i \neq j$. In this situation, we consider the configuration graph $\mathcal{G}(p)$ (resp. the total configuration graph $\mathcal{G}^{\prime}(p ; f)$ ) as follows:

To each divisor $E_{\imath}$, we give a vertex $v_{\imath}$ and a vertex $w$, to each strict transform $\tilde{C}_{\jmath}$. Two vertices are joined by an edge if the corresponding divisors has non-empty intersection. The graph obtained in this way is the total configuration graph $\mathcal{G}^{\prime}(p ; f)$. The graph $\mathcal{G}(p)$ is simply defined by the subgraph with vertices $v_{i} ; 1 \leqq i \leqq s$. It is well known that $\mathcal{G}^{\prime}(p ; f)$ and $\mathcal{G}(p)$ are trees. If $p: Y \rightarrow V$ is a minimal resolution of $f$, the configuration graph $\mathcal{G}(p)$ coincides with the resolution graph defined in $\S 2$. Let $\delta\left(v_{i}\right)$ (resp. $\left.\delta^{\prime}\left(v_{\imath}\right)\right)$ be the number of edges meeting at the vertex $v_{\imath}$ in $\mathcal{G}(p)$ (resp. in $\mathcal{G}^{\prime}(p ; f)$ ). We define the complexity of $p: Y \rightarrow V$ by the complexity of $\mathcal{G}(p)$ :

$$
\rho(p)=1+\sum_{\imath=1}^{s} \max \left(\delta\left(v_{\imath}\right)-2,0\right) .
$$

We say that the modification $p: Y-V$ is quast-effectıve for $f$ if it satisfies the following condition.

(a) $p^{*} f$ has a normal crossing singularity at any non-empty intersections $E_{\imath} \cap E$, with $i \neq \jmath$.

(b) The self-intersection number $E_{\imath}^{2} \leqq-2$ for any $E_{\imath}$ with $\delta^{\prime}\left(v_{\imath}\right) \leqq 2$.

In the case of $p$ being a resolution, (b) implies the minimality. A quasi-effective modification is called effective if the following condition (c) is also satisfied.

(c) $p^{*} f$ has at most normal crossing singularities on each end component $E_{2}$ (i.e., $E_{\imath}$ with $\delta\left(v_{\imath}\right)=1$ ).

LEMMA (3.6). Assume that $p: Y-V$ is quast-effective and assume also that there is a vertex $v_{\imath}$ with $\delta^{\prime}\left(v_{\imath}\right)>2$. Let $m_{\imath}$ be the multiplicity of the function $p^{*} f$ along $E_{\imath}$. Then we have the following inequality:

$$
\sum_{\imath=1}^{s} m_{i}\left(2-\delta^{\prime}\left(v_{\imath}\right)\right)<0
$$

Remark. When $p: Y \rightarrow V$ is a resolution of $f$, the sum $\sum_{\imath=1}^{s} m_{i}\left(2-\delta^{\prime}\left(v_{\imath}\right)\right)$ is equal to the Euler characteristic of the Milnor fiber of $f$ at $O$. 
Proof. Note that any two vertices of $\mathcal{G}(p)$ can be uniquely joined by a finite edges as the graph is a tree. We decompose the graph $\mathcal{G}(p)$ into a finite star graphs in the following way. Let

$$
\left.\left.\mathcal{S}=\left\{v_{i} \in V(\mathcal{Q}) p\right)\right) ; \boldsymbol{\delta}^{\prime}\left(v_{\imath}\right) \geqq 3\right\}
$$

Let $v_{i} \in \mathcal{S}$. Assume that a vertex $v_{j}$ with $\delta^{\prime}\left(v_{j}\right)=1$ can be joined to $v_{\imath}$ without passing any other vertices $v_{k} \in \mathcal{S}$, then we add this path to $v_{\imath}$. The union of such paths make a star with center $v_{i}$. We denote this subgraph by $\mathcal{G}\left(v_{\imath}\right)$. It is immediate by definition that

(1) $\mathcal{G}\left(v_{i}\right) \cap \mathcal{G}\left(v_{k}\right)=\emptyset$ if $v_{i} \neq v_{k}$ and

(2) $\mathscr{B}:=\mathcal{G}(p)-\cup_{v_{i} \in \mathcal{S}} \mathcal{G}\left(v_{i}\right)$ is a disjoint union of bamboos.

For any vertex $v_{l}$ of $\mathscr{B}, \delta^{\prime}(l)=2$ and it does not contribute the sum which we are considering in Lemma (3.6). Thus we can ignore the vertices of $\mathscr{B}$ for our purpose. We will first show that

$$
\sum_{v_{j} \in \mathcal{Q}\left(v_{i}\right)} m_{\jmath}\left(2-\delta^{\prime}\left(v_{\jmath}\right)\right) \leqq 0 .
$$

We assume that $v_{a} \in \mathcal{S}$ and assume that $\mathcal{G}\left(v_{a}\right)$ has $r$-branches of respective length $\mu_{1}, \cdots, \mu_{r}$ in $\mathcal{G}\left(v_{a}\right)$. We renumber each vertex from the center as $v_{1,1}, \cdots$, $v_{1, \mu_{1}} ; \cdots ; v_{r, 1}, \cdots, v_{r, \mu_{r}}$ and we write the corresponding component by $E_{\imath, \jmath}$, $1 \leqq i \leqq r, 1 \leqq j \leqq \mu_{\imath}$. See Figure (3.A).

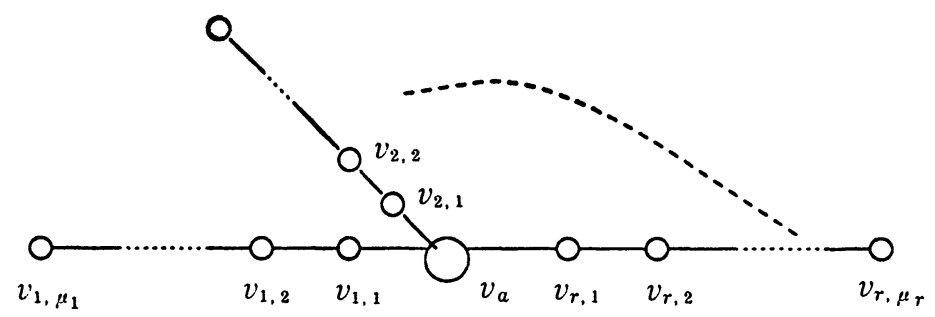

Figure (3.A)

We denote the corresponding coefficient of $E_{\imath, \jmath}$ in $\left(p^{*} f\right)$ by $m_{\imath, \jmath}$. Let

$$
\left(p^{*} f\right)=\sum_{\imath=1}^{r} \sum_{j=1}^{\mu} r m_{\imath, \jmath} E_{\imath, j}+m_{a} E_{a}+D
$$

where $D$ is a linear sum of the other components. Let $l=\delta^{\prime}\left(v_{a}\right)-r$. Note that $l>0$. In fact, if $\mathcal{G}(p)=\mathcal{G}\left(v_{a}\right)$, the vertex $v_{a}$ must intersect with some noncompact vertex $w_{\jmath}$. If $\mathcal{G}(p) \supsetneq \mathcal{G}\left(v_{a}\right)$, the vertex $v_{a}$ must intersect with some compact vertex $v_{\jmath}$, as $\mathcal{G}(p)$ is connected. In any case, we have $r=\delta\left(v_{a}\right)<\delta^{\prime}\left(v_{a}\right)$. Then the sum which we are considering is 


$$
\sum_{v_{j} \in \mathcal{G}\left(v_{a}\right)} m_{j}\left(2-\delta^{\prime}\left(v_{j}\right)\right)=\sum_{i=1}^{r} m_{\imath, \mu_{i}}+(2-r-l) m_{a}
$$

The intersection multiplicities of $E_{\imath, \jmath}$ and $E_{a}$ are determined from the equality:

$$
\begin{aligned}
& 0=\left(p^{*} f\right) \cdot E_{\imath, \jmath}=m_{\imath, j} E_{\imath, j}^{2}+\left(m_{\imath, \jmath-1}+m_{\imath, \jmath+1}\right) \\
& 0=\left(p^{*} f\right) \cdot E_{a}=\sum_{\imath=1}^{r} m_{\imath, 1}+E_{a} \cdot D
\end{aligned}
$$

where $m_{\imath, 0}=m_{a}, m_{\imath, \mu_{i}+1}=0$. As $E_{\imath, \jmath}^{2} \leqq-2$ for $1 \leqq j \leqq \mu_{\imath}$ by the assumption (b), we obtain

$$
2 m_{\imath, \mu_{i}} \leqq m_{\imath, \mu_{i-1}}<m_{\imath, \mu_{i-2}}<\cdots<m_{\imath, 0}=m_{a}
$$

Assume first $r=0$. Then the above sum is obviously negative as $2-\delta^{\prime}\left(v_{a}\right)<0$. Assume next that $r=1$. Then $l \geqq 2$ as $\delta^{\prime}\left(v_{a}\right) \geqq 3$ and by (3.6.2)

$$
\sum_{\imath=1}^{r} m_{\imath, \mu_{i}}+(2-r-l) m_{a}=m_{1, \mu_{1}}+(1-l) m_{a} \leqq m_{1, \mu_{1}}-m_{a}<0
$$

Assume that $r=2$. By (3.6.2), we have $m_{a} \geqq m_{1, \mu_{1}}+m_{2, \mu_{2}}$. Thus we have

$$
\sum_{\imath=1}^{r} m_{\imath, \mu_{i}}+(2-r-l) m_{a} \leqq m_{1, \mu_{1}}+m_{2, \mu_{2}}-m_{a} \leqq 0
$$

If the equality holds, we must have $\mu_{1}=\mu_{2}=1, l=1$ and $m_{a}=2 m_{1,1}=2 m_{2,1}$. If this is the case, we have

$$
E_{a}^{2}=-\frac{m_{1,1}+m_{2,1}+E_{1} \cdot D}{m_{a}}<-1
$$

Now we consider the case that $r \geqq 3$. The $(2-r-l) \leqq-(r-1)$ and

$$
\begin{aligned}
\sum_{\imath=1}^{r} m_{\imath, \mu_{i}}+(2-r-l) m_{a} & \leqq\left(m_{1, \mu_{1}}+m_{2, \mu_{2}}-m_{a}\right)+\left(m_{3, \mu_{3}}+\cdots+m_{r, \mu_{r}}-(r-2) m_{\imath l}\right) \\
& <0 \quad \text { by }(3.6 .2)
\end{aligned}
$$

Thus we have proved

$$
\sum_{i=1}^{s} m_{i}\left(2-\delta^{\prime}\left(v_{i}\right)\right)=\sum_{v_{i} \in S} \sum_{v_{j} \in V\left(\underline{G}\left(v_{i}\right)\right)} m_{j}\left(2-\delta^{\prime}\left(v_{j}\right)\right) \leqq 0
$$

If the equality holds, we have that $E_{i}^{2}<-1$ for any $v_{i} \in S$. As $E_{l}^{2} \leqq-2$ for any $v_{l} \in \mathscr{B}$ by the quasi-effectiveness, we get a contradiction to Fact (3.5). Q. E. D.

Now let us recall the Milnor fibration. Let $f(x, y)$ be a germ of an analytic function with $f(0,0)=0$. Then there exist small positive numbers $\varepsilon$ and $\delta$ $(0<\delta \ll \varepsilon)$ such that $f: E(\varepsilon, \delta) \rightarrow S_{\delta}$ is a local trivial fibration where

$$
E(\varepsilon, \delta):=\left\{(x, y) ;|f(x, y)|=\delta,|x|^{2}+|y|^{2} \leqq \varepsilon^{2}\right\}, \quad S_{\delta}:=\{\eta \in C ;|\eta|=\delta\}
$$


This is called the Milnor fibration of $f(x, y)$ at the origin $O$. We denote the fiber by $F(f ; O)$ and its Euler characteristic by $\chi(f ; O)$. It is well-known that the isomorphism class of the Milnor fibration does not depend on either the coordinates $(x, y)$ or the positive numbers $\varepsilon$ and $\delta$ if they are sufficiently small and $0<\delta \ll \varepsilon$.

COROLlaRY (3.7). (1) Under the same assumption as in Lemma (3.6), we have

$$
\chi(f ; O)=\sum_{a=1}^{t} \chi\left(p^{*} f ; \xi_{a}\right)+\sum_{\imath=1}^{s} m_{i}\left(2-\delta^{\prime}\left(v_{\imath}\right)\right)
$$

where $p^{-1}(O) \cap C=\left\{\xi_{1}, \cdots, \xi_{t}\right\}$. In particular, we have

$$
\chi\left(p * f ; \xi_{\imath}\right)>\chi(f ; O)
$$

(2) The Euler-Poincaré characteristıc $\chi(f ; O)$ is positive if and only if $f=f_{0}^{a}$ for some function germ $f_{0}$ which is non-singular at the origin. In this case, we have $\chi(f ; O)=a$ and we say that $f$ is quasi-smooth. If $f$ is not quasi-smooth, we have $\chi(f ; O) \leqq 0$ and the equality holds if and only if the singularity is a normal crossing singularity at $O$.

Proof. Let $\eta_{2, l}$ be the intersection of $E_{i} \cap E_{l}$ (if not empty) and fix a coordinate system $\left(x_{a}, y_{a}\right)$ and $\left(x_{i, j}^{\prime}, y_{i, j}^{\prime}\right)$ centered at $\xi_{a}$ and $\eta_{i, j}$ respectively for $a=1, \cdots, t, i \neq j$. Let $B_{\imath, \jmath}$ and $B_{a}$ be the ball of radius $\varepsilon$ centered at $\eta_{2,3}$ and $\xi_{a}$ respectively:

$$
\begin{aligned}
B_{\imath, \jmath} & =\left\{\left(x_{\imath, \jmath}, y_{\imath, j}\right) ;\left|x_{\imath, \jmath}\right|^{2}+\left|y_{\imath, j}\right|^{2} \leqq \varepsilon^{2}\right\} \\
B_{a} & =\left\{\left(x_{\imath}, y_{\imath}\right) ;\left|x_{\imath}\right|^{2}+\left|y_{\imath}\right|^{2} \leqq \varepsilon^{2}\right\}
\end{aligned}
$$

We also fix a metric on $X$. Let $U$ be the $\varepsilon^{\prime}$-neighborhood of $\pi^{-1}(O)$. Let $W=$ $U \cup_{\imath, j} B_{\imath, j} \cup_{a} B_{a}$ and let $\tilde{F}=\left(p^{*} f\right)^{-1}(\delta) \cap W=\{Q \in W ; f(\pi(Q))=\delta\}$ and let $F_{\imath, j}=$ $\widetilde{F} \cap B_{\imath, \jmath}$ and $F_{a}=\widetilde{F} \cap B_{a}$. Let $\left(p^{*} f\right)=\sum_{\imath=1}^{s} m_{i} E_{i}+\sum_{a=1}^{t} C_{a}$. Here $m_{\imath}$ is the multiplicity of the function $p^{*} f$ along $E_{\imath}$. If $\varepsilon, \varepsilon^{\prime}, \delta$ are sufficiently small and $0<\delta \ll \varepsilon^{\prime} \ll \varepsilon$, by a standard argument we see that

(1) $\tilde{F}$ is diffeomorphic to $F(f ; O)$,

(2) $F_{\imath, \jmath}$ and $F_{a}$ are diffeomorphic to the respective Milnor fiber $F\left(p^{*} f ; \eta_{\imath, j}\right)$ and $F\left(p^{*} f ; \xi_{a}\right)$.

(3) The complement $\tilde{F}-\cup_{\imath, j} F_{\imath, j}-\cup_{a} F_{a}$ is disjoint union of s-components, say $G_{\imath}, i=1, \cdots, s$, and $G_{\imath}$ is diffeomorphic to a $m_{i}$-fold covering space over $E_{\imath}^{\prime}$ using the projection map $p_{\imath}$ of a tubular neighborhood of $E_{\imath}$ :

$$
p_{\imath}: G_{\imath} \stackrel{\text { m.fold }}{\longrightarrow} E_{\imath}^{\prime}, \quad E_{\imath}^{\prime}=E_{\imath}-E_{i} \cap\left(\cup_{\jmath \neq \imath} B_{\imath, j} \cup_{\xi_{a} \in E_{\imath}} B_{a}\right)
$$

By the definition of $\delta^{\prime}\left(v_{i}\right)$, this space is homeomorphic to $\boldsymbol{P}^{1}$ minus $\delta^{\prime}\left(v_{i}\right)$ disks. From the decomposition $\tilde{F}=\cup_{\imath \jmath} F_{2 j} \cup_{a} F_{a} \cup_{\imath} G_{\imath}$ and the additivity of EulerPoincaré characteristics, the equality (3.7.1): 


$$
\chi(f ; O)=\sum_{a} \chi\left(\pi^{*} ; \xi_{a}\right)+\sum_{i} m_{i}\left(2-\delta^{\prime}\left(v_{i}\right)\right)
$$

follows immediately. Here we have used the following facts:

(4) $\chi\left(p^{*} f ; F_{\imath, j}\right)=0$ as $p^{*} f$ has an normal crossing singularity at $\eta_{\imath, j}$.

(5) $\chi\left(G_{\imath}\right)=m_{i}\left(2-\delta^{\prime}\left(v_{i}\right)\right)$ by (3).

(6) The Euler-Poincaré characteristics of the various intersections $G_{i} \cap F_{a}$, or $G_{i} \cap F_{\imath, \jmath}$ of the above decomposition are zero as they are disjoint sums of $S^{1}$.

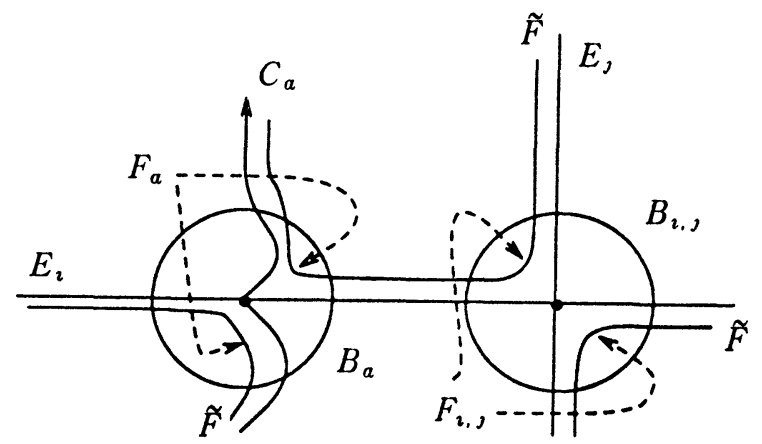

Figure (3.B)

Now we prove the assertion (2) before showing (3.7.2). Assume that $p: Y \rightarrow V$ is a minimal resolution of $f$. Assume that $\mathcal{G}^{\prime}(p ; f)$ is a bamboo i. e., $\delta^{\prime}\left(v_{i}\right) \leqq 2$ for any $i=1, \cdots, s$. Then $\delta\left(v_{i}\right)^{\prime} \leqq 2$ and by the minimality this is the case only when $s=0$. This implies either (i) $f=f_{0}^{a}$ for some $a>0$ and $f_{0}$ being nonsingular or (ii) $f$ has a normal crossing singularity at the origin. In these cases, we have $\chi(f ; O)=a$ or $\chi(f ; O)=0$ respectively. See [O1] for the second equality. Assume that the singularity of $f$ at the origin is neither quasi-smooth nor a normal crossing singularity. Then we are in the situation as in Lemma (3.6). Note that $p^{*} f$ has a normal crossing singularity at $\xi_{a}, 1 \leqq a \leqq s$ as $p$ is assumed to be a minimal resolution. Thus $\chi\left(p^{*} f ; \xi_{a}\right)=0$ and the inequality (3.7.2) gives

$$
\begin{aligned}
\chi(f ; O) & =\sum_{a} \chi\left(p^{*} f ; \xi_{a}\right)+\sum_{\imath} m_{i}\left(2-\delta^{\prime}\left(v_{\imath}\right)\right) \\
& =\sum_{\imath} m_{i}\left(2-\delta^{\prime}\left(v_{i}\right)\right)<0 \quad \text { by Lemma (3.6). }
\end{aligned}
$$

This proves the assertion (2). We come back to the situation as in (1) where $p$ is a quasi-effective modification. The inequality (3.7.2) follows immediately from Lemma (3.6), (3.7.1) and (2):

$$
\chi(f ; O)=\sum_{a} \chi\left(p^{*} f ; \xi_{a}\right)+\sum_{i} m_{i}\left(2-\delta^{\prime}\left(v_{i}\right)\right)<\chi\left(p^{*} f ; \xi_{a}\right)
$$

This completes the proof. Q.E.D. 
COROLLARY (3.8). Assume that the singularity of $f$ at the origin is neither quasi-smooth nor a normal crossing singularity. Let $(x, y)$ be a quasi-good coordinates and let $\pi: X \rightarrow V$ be the associated toroudal modification. Then $\pi$ is quasieffective and under the same notation as in $\S 2, \chi\left(\pi * f ; \xi_{\imath, j}\right)>\chi(f ; O)$.

Proof. The quasi-effectiveness follows from Lemma (2.12) and the property $\hat{E}\left(P_{\imath, j}\right)^{2} \leqq-2$ for any $0 \leqq i \leqq m, 1 \leqq \jmath \leqq l_{\imath}$.

COROLLARY (3.9). For any function germ $f$, there exists a quasi-good (resp. good) toroidal resolution $q: Z \rightarrow V$ of $f$.

Proof. Consider first a quasi-good (resp. good) toroidal blowing-up at the origin $\pi_{1}: X_{1} \rightarrow V$. If $\pi^{*} f$ has still some singularity $\xi_{2,}$, which is different from a normal crossing singularity, we take an admissible quasi-good (resp. good) coordinate centered at $\xi_{\imath, 3}$ by Lemma (1.3) or Lemma (1.7). The number of such $\xi_{\imath, j}$ 's is not bigger than $-\chi(f ; O)$ by Corollary (3.7). Then we take the associated blowing-up at $\xi_{2,}$ and continue the same operation on the upstairs if necessary. Each tower of the singularities has at most $-\chi(f ; O)$ steps again by Corollary (3.7). Q.E. D.

Now we study how the configuration graph and the complexity of the modification change under the composition of the modifications.

Let $f$ be as in $\S 2$ and let $\pi: X \rightarrow V$ be the associated toroidal modification with respect to the system of coordinates $(x, y)$. We use the same notations as in $\S 2$. Recall that we have shown in $\S 2$ :

Proposition (3.10). (i) The graph of $\pi$ is a bamboo with $m+\sum_{\imath=0}^{m} l_{i}$-vertices $v_{0,1}, \cdots, v_{m, l_{m}}$ corresponding to the exceptional divisor $\hat{E}\left(P_{0,1}\right), \cdots, \hat{E}\left(P_{m, l_{m}}\right) .\left(P_{0,1}\right.$ $=P_{1}$ if $l_{0}=0$ and $P_{m, l_{m}}=P_{m}$ if $l_{m}=0$.) The non-compact divisor $\hat{E}\left(P_{0}\right)$ (resp. $\hat{E}\left(P_{m+1}\right)$ which is the pull back of $x=0$ (resp. $y=0$ ) intersects only with $\hat{E}\left(P_{0,1}\right)$ (resp. $\left.\hat{E}\left(P_{m, l_{m}}\right)\right)$.

We have $\hat{E}\left(P_{\imath, j}\right)^{2} \leqq-2$ for $0 \leqq i \leqq m, 1 \leqq \jmath \leqq l_{\imath}$ and $\delta^{\prime}\left(v_{\imath, 0}\right) \geqq 3$ for $2 \leqq \imath \leqq m-1$.

(ii) If $x$ (resp. $y)$ is quasi-good for $f$, either $l_{0} \neq 0$ or $l_{0}=0$ and $\delta^{\prime}\left(v_{1,0}\right) \geqq 3$ (resp. $l_{m} \neq 0$ or $l_{m}=0$ and $\delta^{\prime}\left(v_{m, 0}\right) \geqq 3$.

(iii) If $x$ (resp. $y$ ) is good, the function $\pi^{*} f$ has at most normal crossing singularities on the left end divisor $\hat{E}\left(P_{0,1}\right)$ (resp. the right end divisor $\hat{E}\left(P_{m, l_{m}}\right)$ ). (iv) In particular, the quasi-good (resp. good) torondal blowing-up is quasi-effective (resp. effective).

The assertion (iii) follows from Lemma (2.12). Now we consider the composition of modifications.

LEMMA (3.11). (i) Let $p: Y \rightarrow V$ be an quast-effective modification for $f$ at the origin and let $\tilde{C}$ be the proper transform of $C=\{f=0\}$. Let $E_{1}, \cdots, E_{s}$ be the components of $p^{-1}(O)$ and let $\xi \in E_{i} \cap \tilde{C}$ be a singular point of $p^{*} f$ which is 
not a normal crossing singularity. Let $(u, v)$ be an admissible quasi-good (resp. good) coordinate for $p^{*} f$ at $\xi$ and let $\pi_{1}: Y_{1} \rightarrow Y$ be the associated torordal blowingup. (We assume that $E_{\imath}$ is defined by $u=0$.) Then the composition $p_{1}: p \circ \pi_{1}: Y_{1}$ $\rightarrow V$ is a quasi-effective modification and the configuration graph $\mathcal{G}\left(p_{1}\right)$ is obtained from the disjoint sum of $\mathcal{G}(p)$ and $\mathcal{G}\left(\pi_{1}\right)$ by joining the vertex $v_{\imath}$ and the left end vertex of $\mathcal{G}\left(\pi_{1}\right)$.

(ii) Furthermore in the case that $p$ is effective and $(u, v)$ is admissible good, we have the equality: $\rho\left(p_{1}\right)=\rho(p)+1$.

Proof. We know that $\mathcal{G}\left(\pi_{1}\right)$ is a bamboo. Let $F_{1}, \cdots, F_{t}$ be the components of $\pi_{1}^{-1}(\xi)$ from the left and let $v_{1}^{\prime}, \cdots, v_{t}^{\prime}$ be the corresponding vertices of $\mathcal{G}\left(\pi_{1}\right)$. Let $E_{\jmath}^{\prime}$ be the proper transform of $E_{\jmath}(1 \leqq \jmath \leqq s)$ respectively. It is obvious that $E_{1}^{\prime}, \cdots, E_{s}^{\prime}$ and $F_{1}, \cdots, F_{t}$ are the components of $p_{1}^{-1}(O)$. As $\pi_{1}\left(F_{a}\right)$ $=\{\xi\}$ and $\xi \notin E$, for $j \neq i$ by the quasi-effectivity of $p, E_{j}^{\prime} \cap F_{a}=\emptyset$. As $E_{\imath}$ corresponds to $\{u=0\}, E_{\imath}^{\prime}$ intersects with $F_{1}$. Thus the configuration graph of $p_{1}$ is simply obtained by adding the bamboo $\mathcal{G}\left(\pi_{1}\right)$ at the vertex $v_{i}$ corresponding to $E_{\imath}$. We assert $E_{\jmath}^{\prime 2}=E_{\jmath}^{2}, j \neq i$ and $E_{\imath}^{\prime 2}<E_{\imath}^{2}$. The first equality is obvious and the second equality follows from the well-known fact that (i) $\pi_{1}$ is a finite composition of ordinary blowing-ups and under an ordinary blowing-up whose center is on a compact smooth divisor $D$, the self-intersection number of the proper transform $D^{\prime}$ of $D$ goes down by 1 (see for instance [La] Lemma (4.3) or [04]). Thus $p_{1}$ is quasi-effective. This proves the assertion for the case that $p$ is quasi-effective and $\pi_{1}$ is admissible quasi-good toroidal modification. Assume that $p$ is effective and $(u, v)$ is admissible good. By the effectiveness, $p^{*} f$ has at most normal crossing singularities at each end divisor $E$, of $\mathcal{G}(p)$ (i. e., $\left.\delta_{\jmath}=1\right)$. In particular, $E_{\imath}$ is not an end divisor. Thus we obtain the equality: $\rho\left(p_{1}\right)=\rho(p)+1$. By Proposition (3.10) and the above observation, $p_{1}^{*} f$ has at most normal crossing singularities on $E_{\jmath}^{\prime}$ with $\delta_{\jmath}=1$ and on the right end divisor $F_{t}$ of $\mathcal{G}\left(\pi_{1}\right)$. Thus $p_{1}$ is effective. Q. E. D.

Now we are ready to state our main theorem which is an immediate consequence of Lemma (3.11).

THEOREM (3.12). (i) Assume that $\pi_{1} \circ \cdots \circ \pi_{k}$ is a quasi-good toroidal resolution. Then $\pi_{1} \circ \cdots \circ \pi_{k}$ gives a minimal resolution of $f$.

(ii) Assume that $\pi_{1} \circ \cdots \circ \pi_{k}$ is a good toroidal resolution. Then the number of good toroidal blowing-ups $k$ is equals to the resolution complexity of $f: k=\rho(f)$. In particular, the number of good toroidal blowing-ups does not depend on the choice of the good toroidal blowing-ups.

As an application of Theorem (3.12), we obtain:

COROLLARY (3.13). Let $f$ be a given function.

(i) The quasi non-degeneracy does not depend on the choice of a good system of coordinates. 
(ii) Assume that $f$ is reduced. Then the non-degeneracy does not depend on the choice of a good system of coordinates.

Proof. Assume that $(x, y)$ is a good system of coordinates for $f$. Then by Theorem (3.12), $\rho(f)=1$ if and only if $f$ is quasi non-degenerate in $(x, y)$. This proves (i). Assume that $f(x, y)$ is non-degenerate in a good system of coordinates. Then $f$ is also quasi non-degenerate. Thus $f$ is quasi nondegenerate in any good system of coordinates. Assume that $(u, v)$ be a good system of coordinates. Let $f^{\prime}(u, v):=f(x(u, v), y(u, v))$. Then $f^{\prime}(u, v)$ is quasi non-degenerate. By the definition of quasi non-degeneracy, for reduced function germ, $f^{\prime}(u, v)$ is quasi non-degenerate if and only if $f^{\prime}(u, v)$ is non-degenerate.

\section{Remarks}

In this section, we give a few remarks

\section{(I) Non-admissible good coordinates}

Assume that we have chosen a finite admissible good toroidal blowing-up

$$
X_{s} \stackrel{\pi_{s}}{\longrightarrow} X_{s-1} \stackrel{\pi_{s-1}}{\longrightarrow} \cdots \longrightarrow X_{1} \stackrel{\pi_{1}}{\longrightarrow} C^{2}
$$

Let $p_{s}=\pi_{1} \circ \cdots \circ \pi_{s}: X_{s} \rightarrow C^{2}$ and let $E_{1}, \cdots, E_{t}$ be components of $p_{s}^{-1}(O)$. Let $f_{s}:=p_{s}^{*} f$. Let $\xi \in\left(E_{i}-\cup_{\jmath \neq \imath} E_{\jmath}\right)$ and assume that $f_{s}$ has a singularity at $\xi$ which is not an ordinary double point. As we have seen in $\S 3$, we can take an admissible good system of coordinates $\left(x_{1}, y_{1}\right)$ centered at $\xi$ such that $x_{1}=0$ defines $E_{\imath}$. The coordinate $y_{1}$ is good by definition but the coordinate $x_{1}$ may not good, though it is quasi-good of course. Assume that $x_{1}$ is not good. Let $Q_{1}, \cdots, Q_{m}$ be the covectors corresponding to the faces of $\Gamma\left(f_{s} ; x_{1}, y_{1}\right)$ and let

$$
f_{s, Q_{1}}=b_{1} x_{1}^{r_{1}} y_{1}^{s_{1}} \prod_{\imath=1}^{k_{1}}\left(x_{1}-\gamma_{j} y_{1}^{a_{1}}\right)^{\nu_{j}}, \quad Q_{1}={ }^{t}\left(a_{1}, 1\right)
$$

Then there exists good coordinate system $\left(x_{2}, y_{2}\right)$ so that

$$
y_{1}=y_{2}, \quad x_{1}=x_{2}+\gamma_{\rho_{0}} y_{2}^{a_{1}}+h\left(x_{2}, y_{2}\right) \quad \text { for some } 1 \leqq j_{0} \leqq k_{1}
$$

Note that $\left(x_{2}, y_{2}\right)$ is not an admissible system of coordinates in the sense of Definition (3.4). Now the face $\Delta\left(Q_{1} ; f_{s}\right)$ breaks into $q$ faces $(q \geqq 2)$ and $f_{s, Q_{1}}$ changes as

$$
c_{1}\left(x_{2}+\gamma_{j} y_{2}^{a_{1}}\right)^{r_{1}} y_{2}^{s_{1}} x_{2}^{\nu_{j} j_{0}} \prod_{j \neq j_{0}}\left(x_{2}-\left(\gamma_{j}-\gamma_{j_{0}}\right) y_{2}^{a_{1}}\right)^{\nu_{j}}
$$

Let $p: Y \rightarrow X_{s}$ be the toroidal blowing-up with respect to $\Gamma^{*}\left(f_{s} ; x_{2}, y_{2}\right)$. As $x_{1}^{r_{1}}$ divides $f_{s},\left(x_{2}+\gamma_{3} y_{2}^{a_{1}}\right)^{r_{1}}$ also divide $f_{s}\left(x_{2}+\gamma_{s_{0}} y_{2}^{a_{1}}+h\left(x_{2}, y_{2}\right), y_{2}\right)$. Thus $p^{*} f_{s}$ has a normal crossing singularity near the divisor $\tilde{E}_{\imath}$ which is the proper transform of $E_{i}=\left\{x_{1}=0\right\}$ (Lemma (2.8)). Let $p^{\prime}=p_{s^{\circ}} p: Y \rightarrow C^{2}$. Now the configuration graph $\Gamma\left(p^{\prime}\right)$ is simply obtained by joining the configuration graph $\Gamma\left(p_{s}\right)$ and 
$\Gamma(p)$ at the vertices corresponding to $E_{\imath}$ and $\hat{E}\left(Q_{1}\right)$. See Figure (4.1.A). Now it is easy to see that

$$
\rho\left(p^{\prime}\right)=\rho\left(p_{s}\right)+2
$$

Thus the above non-admissible good toroidal blowing-up is the composition of two admissible blowing-ups.

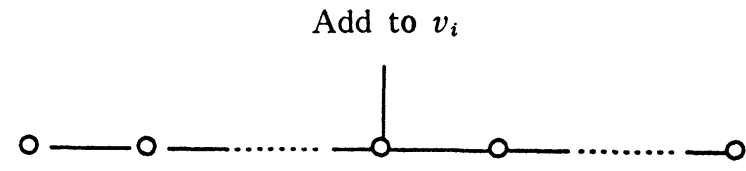

Figure (4.1.A)

(II) Finiteness of admissible blowing-ups

To show the finiteness of admissible blowing-ups, we can also use the following argument. Let $f(x, y)$ be as in the section 1 . We use the same notation as in the section 1 or 2 . We define the level of degeneracy $\eta(f ; x, y)$ of $f$ relatively to the system of coordinates $x, y$ by the following:

$$
\eta(f ; x, y)=\max _{1 \leqq i \geqq m, 1 \leqq \jmath \leqq k_{\imath}}\left(\nu_{i, j}\right) .
$$

$(\eta(f ; x, y)$ is assumed to be 0 if $m=0)$. Notice that $f$ is non-degenerate if and only if $\eta(f ; x, y)=1$ or 0 .

Then we have the following simple lemma.

Lemma (4.2). Let $f$ be as above. Suppose that $(x, y)$ is a system of quasigood coordinates for $f$ at the point $O$. Let $\pi$ be the good toroidal blowing-up of $f$ in these coordinates and suppose it is a non-trivial toroidal blowing-up. Let $\xi_{1}$ be a point in the component $\hat{E}(P)$ of $\pi^{-1}(O)$ where the total transform of $f$ has not normal crossings. Let $\left(x_{1}, y_{1}\right)$ be an admissible quasi-good coordinates for the total transform $\pi^{*} f$ of $f$ at the point $\xi_{1}$. Then we have:

$$
\eta(f ; O ; x, y)>\eta\left(\pi^{*} f ; \xi_{1} ; x_{1}, y_{1}\right)
$$

This inequality may not hold if we take non-admissible good coordinate system.

(III) A toroidal blowing-up is a finite composition of blowing-ups

We have remarked already that a toroidal blowing-up is a composition of a sequence of blowing-ups. This fact can be also proved quite elementarily as follows

Proposition (4.3). Any toroidal blowing-up is a sequence of point blowingups. 
Proof. We assume that we start for the point $O$ which is the origin. Let $\Sigma^{*}$ be a regular subdivision of the dual diagram $\left[R_{-\infty}, R_{0}\right]$. Let $\pi: X \rightarrow C^{2}$ be the corresponding toroidal blowing-up.

First we notice that, if $\Sigma^{*}$ is non-trivial, the vertex ${ }^{t}(1,1)$ is among the vertices of $\Sigma^{*}$. In $\Sigma^{*}$ there is a unique pair of vertices $(P, Q)$ such that $(P, Q)$ is a simplex of $\Sigma^{*}$ and, for $P={ }^{t}(a, b)$ and $Q={ }^{t}\left(a^{\prime}, b^{\prime}\right), a \geqq b$ and $a^{\prime}<b^{\prime}$. Because $\Sigma^{*}$ is regular, we have $a b^{\prime}-b a^{\prime}=1$ and

$$
1=a b^{\prime}-b a^{\prime} \geqq b\left(b^{\prime}-a^{\prime}\right) \geqq 0
$$

If $b=0$, we must have $a=b^{\prime}=1$ and then $(P, Q)=\left(R_{-\infty}, R_{0}\right)$ which contradicts to the assumption. So $b>0$ and we have necessarily that $b=b^{\prime}-a^{\prime}=1$ and $a=b$. Thus $P={ }^{t}(1,1)$.

Now consider the blowing-up $e: Y \rightarrow C^{2}$ of the point $O$. We have two charts $U_{1}$ and $U_{2}$ with coordinates $(s, t)$ and $(u, v)$. The blowing-up $e$ is given by $e_{1}$ and $e_{2}$ given by:

$$
\begin{array}{ll}
x=s t, & y=t \\
x=u, & y=u v
\end{array}
$$

This is the toroidal blowing-up with respect to the simplest non-trivial regular subdivision with three vertices $\left\{R_{\infty}, P, R_{0}\right\}$ where $P={ }^{t}(1,1)$. If non-trivial, the regular subdivision $\Sigma^{*}$ gives two subdivisions $\Sigma_{1}$ and $\Sigma_{2}$ ending and starting by $P={ }^{t}(1,1)$ respectively. These subdivisions define subdivisions $\hat{\Sigma}_{1}$ and $\hat{\Sigma}_{2}$ of $\left[R_{-\infty}, R_{0}\right]$ by the corresponding affine transformations

$$
\begin{aligned}
& \Sigma_{1} \longrightarrow \hat{\Sigma}_{1}, \quad{ }^{t}(\alpha, \beta) \longmapsto{ }^{t}(\alpha-\beta, \beta) \\
& \Sigma_{2} \longrightarrow \hat{\Sigma}_{2}, \quad{ }^{t}(\alpha, \beta) \longmapsto{ }^{t}(\alpha, \beta-\alpha)
\end{aligned}
$$

in which $P$ is respectively sent onto $R_{0}$ and $R_{\infty}$. It is obvious that these subdivisions $\hat{\Sigma}_{1}$ and $\hat{\Sigma}_{2}$ are regular. They define two toroidal blowing-ups $\pi_{1}: X_{1} \rightarrow U_{1}$ and $\pi_{2}: X_{2} \rightarrow U_{2}$. The spaces $X_{1}$ and $X_{2}$ are respectively biholomorphic to $U_{1}$ and $U_{2}$ outside the inverse images of the origin and can be glued into a space $X^{\prime}$ and, by gluing the maps $\pi_{1}$ and $\pi_{2}$, we obtain a map $\pi^{\prime}: X^{\prime} \rightarrow Y$. Let us explicit this map. Consider $\hat{\sigma}=\left(\hat{P}_{1}, \hat{P}_{2}\right)$ be a simplex in $\Sigma_{1}$ (resp. $\Sigma_{2}$ ). Write

$$
\left(\hat{P}_{1}, \hat{P}_{2}\right)=\left({ }^{t}\left(\hat{p}_{11}, \hat{p}_{21}\right),{ }^{t}\left(\hat{p}_{12}, \hat{p}_{22}\right)\right)
$$

Then $\pi^{\prime}$ is defined by

$$
\left.s=y_{\sigma, 1}^{\hat{p}_{11}} y_{\sigma, 2}^{\hat{p}_{12},} \quad t=y_{\sigma, 1}^{\hat{p}_{21}} y_{\sigma, 2}^{\hat{p}_{22}} \quad \text { (resp. } u=y_{\sigma, 1}^{\hat{p}_{11}} y_{\sigma, 2}^{\hat{p}_{12}}, v=y_{\sigma, 1}^{\hat{p}_{21}} y_{\sigma, 2}^{\hat{p}_{22}}\right) .
$$

One can check that $X^{\prime}$ is obtained by the charts obtained by composing these charts and $e_{1}$ or $e_{2}$. There is a canonical biholomorphic map of $X^{\prime}$ onto $X$. We define this map from $X_{1}$ (resp. $X_{2}$ ) into $X$ : let $\hat{\sigma}$ in $\hat{\Sigma}_{1}$ (resp. $\hat{\Sigma}_{2}$ ); it defines a simplex $\sigma$ of $\Sigma^{*}$, so the map of $X_{1}$ into $X$ read in the corresponding 
charts is the identity. One checks easily that the corresponding gluings give the biholomorphic isomorphism. So the assertion of the proposition derives from an induction on the number of vertices in the dual diagram.

\section{REFERENCES}

[B] E. Brieskorn And H. KNörrer, Ebene Algebraische Kurven, Birkhäuser, BaselBoston-Stuttgart, 1981.

[K] A.G. Kouchnirenko, Polyèdres de Newton et nombres de Milnor, Invent. Math., 32 (1976), 1-32.

[La] H.B. Laufer, Normal Two-Dimensional Singularities, Ann. of Math. Stud., 71, Princeton Univ. Press, Princeton, 1971.

[L-M-W] D. T. LE, F. Michel AND C. WEBER, Courbes polaires et topologie des courbes planes, Ann. Sci. Ecole Norm. Sup. 4ème Série, 24 (1991), 141-169.

[M] J. MiLnor, Singular Points of Complex Hypersurface, Ann. of Math. Stud., 61, Princeton Univ. Press, Princeton, 1968.

[N] M. Noether, Uber die algebraischen Funktionen einer und zweier Variabeln, Götting. Nachr. (1871), 267-278.

[01] М. ОкА, On the homotopy types of hypersurfaces defined by weighted homogeneous polynomials, Topology, 12 (1973), 19-32.

[O2] M. OKa, On the resolution of two-dimensional singularities, Proc. Japan Acad., 60 (1984), 174-177.

[03] M. OKA, On the resolution of hypersurface singularities, Complex Analytic Singularities, Adv. Stud. Pure Math., 8, North-Holland, 1987, 405-436.

[04] M. OKA, Geometry of plane curves via toroidal resolntion (preprint, to appear in Proceeding of La Rabida Conference 1991).

[V] A.N. VARChENKo, Zeta-function of monodromy and Newton's diagram, Invent. Math., 37 (1976), 253-262.

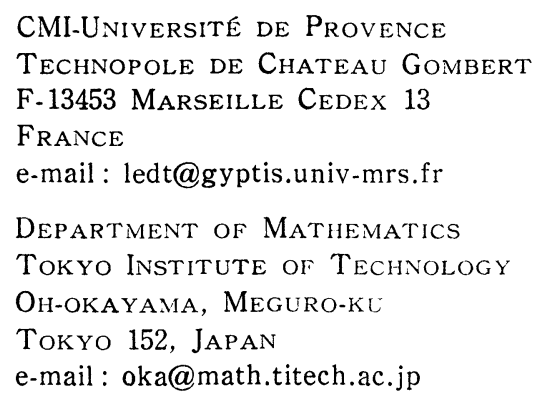

